\title{
The prognostic roles of CYP19A1 expression in bladder cancer patients of different genders
}

\author{
Shanghua Cai ${ }^{1 \#}$, Yuanfa Feng ${ }^{1 \#}$, Jianheng Ye ${ }^{2 \#}$, Yulin Deng ${ }^{1}$, Zhiduan Cai ${ }^{3}$, Xuejin Zhu ${ }^{1}$, Ren Liu ${ }^{2}$, \\ Yixun Zhang ${ }^{2}$, Zhihao Zou ${ }^{2}$, Zhenfeng Tang ${ }^{1}$, Zhaodong Han ${ }^{2}$, Chi Tin Hon ${ }^{4}$, Weide Zhong ${ }^{1,2,5,}$, \\ Huichan $\mathrm{He}^{1}$
}

${ }^{1}$ Department of Urology, Guangdong Key Laboratory of Urology, The First Affiliated Hospital of Guangzhou Medical University, Guangzhou Medical University, Guangzhou, China; ${ }^{2}$ Department of Urology, Guangdong Key Laboratory of Clinical Molecular Medicine and Diagnostics, Guangzhou First People's Hospital, School of Medicine, South China University of Technology, Guangzhou, China; ${ }^{3}$ Department of Urology, The Fifth Affiliated Hospital of Guangzhou Medical University, Guangzhou, China; ${ }^{4}$ Macau Institute of Systems Engineering, Macau University of Science and Technology, Macau, China; ${ }^{5}$ Department of Urology, Huizhou Municipal Central Hospital, Huizhou, China; ${ }^{6}$ Macau Institute for Applied Research in Medicine and Health, Macau University of Science and Technology, Macau, China

Contributions: (I) Conception and design: S Cai, Y Feng, J Ye, H He, W Zhong; (II) Administrative support: W Zhong, Z Han; (III) Provision of study materials or patients: Y Deng, Z Cai, X Zhu, CT Hon; (IV) Collection and assembly of data: R Liu, Y Zhang; (V) Data analysis and interpretation: S Cai, Z Zou, Z Tang; (VI) Manuscript writing: All authors; (VII) Final approval of manuscript: All authors.

\#These authors contributed equally to this work.

Correspondence to: Huichan He. Department of Urology, Guangdong Key Laboratory of Urology, The First Affiliated Hospital of Guangzhou Medical University, Guangzhou Medical University, Guangzhou 510230, China. Email: xiaohejian@21cn.com; Weide Zhong. Department of Urology, Guangdong Key Laboratory of Urology, The First Affiliated Hospital of Guangzhou Medical University, Guangzhou Medical University, Guangzhou 510230, China. Email: zhongwd2009@live.cn.

Background: The incidence of bladder cancer $(\mathrm{BCa})$ in male is approximately three to four times higher than in female, but the oncological outcomes in female patients with BCa are significantly worse than in male patients. Although many biomarkers have been identified in recent decades to predict the prognosis of BCa patients, few of them are able to distinguish the prognosis of BCa patients with gender difference. Aromatase encoded by the CYP19A1 gene catalyzes the conversion of androgens to estrogens. In this study, we investigate the prognosis significance of CYP19A1 expression considering the gender difference in BCa patients from four available public databases.

Methods: Four available public databases of BCa, including GSE13507, TCGA-BLCA, E-MTAB-4321, and E-MTAB-1803, were utilized in this analysis. The overall survival (OS) and progression-free survival (PFS) in different stages and genders were evaluated using the Kaplan-Meier analysis based on the optimal cut-off values of CYP19A1 expression. Then, Gene Set Enrichment Analysis (GSEA) were further performed to explore the potential biologic pathways by altering CYP19A1 expression in BCa patients.

Results: The results showed that patients with high CYP19A1 expression had a poorer outcome compared with those with low expression in both BCa cohorts in general. Higher CYP19A1 expression in male patients were significantly associated with shorter survival for either non-muscle-invasive bladder cancer (NMIBC) or muscle-invasive bladder cancer (MIBC). However, female NMIBC patients with high CYP19A1 expression were identified to have a better prognosis, whereas high CYP19A1 expression in female MIBC patients were significantly associated with poorer survival. The result of the GSEA showed that different outcomes in female and male patients with NMIBC were related to the interaction of CYP19A1 and the cell-cycle-related pathways.

Conclusions: These findings demonstrated that CYP19A1 expression might have a potential role in distinguishing the prognosis of female BCa patients dependent on tumor stage. Our results provide new insights for aromatase-mediated BCa therapy. 
Keywords: CYP19A1; bladder cancer (BCa); gender difference; prognosis; bioinformatics analysis

Submitted May 06, 2021. Accepted for publication Aug 02, 2021.

doi: $10.21037 / \mathrm{tau}-21-400$

View this article at: https://dx.doi.org/10.21037/tau-21-400

\section{Introduction}

According to new global cancer statistics, bladder cancer (BCa) is the 10th most common malignancy worldwide. In addition, the incidence of $\mathrm{BCa}$ in males (424,082 cases) remains three to four times higher than in females (125,311 cases) (1). It was reported that the main $\mathrm{BCa}$ risk factors, e.g., smoking and chemical exposures, are more common in male than in female (2). Still, a study demonstrated this gender difference in incidence is not affected by those BCa exposure risks (3). Besides, approximately $75 \%$ of $\mathrm{BCa}$ patients were diagnosed as non-muscle-invasive bladder cancer (NMIBC), and the rest of them suffered from muscle-invasive bladder cancer (MIBC) (4). Cancer survival statistics reported that NMIBC patients have a better five-year relative survival rate than MIBC patients (5). However, gender difference may also affect the cancer-specific mortality of $\mathrm{BCa}$, and female patients are more likely to have advanced disease (6). A population-based cohort study demonstrated that female MIBC patients had higher cancerspecific mortality than male MIBC patients, and this high mortality in female MIBC patients could not be explained by gender differences in age, grade, clinical stage, and other clinicopathological features (7). In contrast, despite the fact that the male $\mathrm{BCa}$ patients had a higher incidence, they displayed a better survival rate (8). In recent decades, many novel biomarkers have been identified to predict $\mathrm{BCa}$ patient prognosis, but few of them can distinguish the prognosis of male and female BCa patients.

Sex hormones present in the human body are involved in regulating physiological activities. Aromatase encoded by the CYP19A1 gene can catalyze the irreversible conversion of androgens to estrogens (9). Its activity has been observed in both male and female organs (9), and it is abundantly expressed in peripheral tissues, such as plasma and adipose tissue (10-12). Thus, it can be utilized to synthesize estrogens at different physiological stages in males and females. Additionally, aberrant aromatase expression and its promotion effects on tumor progression have been found in various cancers (13-17). Accordingly, the application of aromatase inhibitors for cancer treatment has been well investigated in recent decades, especially in women's cancers (18).

Existing studies have primarily focused on investigating the prognostic role of aromatase in BCa. The positive expression of aromatase was found to be significantly associated with advanced stage and poorer survival of $\mathrm{BCa}$ patients (19). In addition, higher aromatase concentrations in the plasma of MIBC patients compared with those in NMIBC patients were observed (20). In a previous study, $\mathrm{BCa}$ patients with high aromatase expression in the tumorrelated stroma were found to have shorter overall survival (OS) compared with those with low aromatase expression (21). Aromatase was also found to be more frequently expressed in male patients (21).

According to the gender difference in the unique behaviors of $\mathrm{BCa}$, sex hormone may have significant effects on these behaviors (3). To date, aromatase is the only enzyme known to catalyze the irreversible conversion of androgen to estrogen in human beings (22), and the prognostic roles of aromatase in female and male $\mathrm{BCa}$ patients have not yet been elucidated. In this study, we aim to analyze the association between the expression of CYP19A1 (the coding gene of aromatase) and the prognosis of female and male BCa patients in four BCa cohorts obtained from public databases. We present the following article in accordance with the MDAR checklist (available at https://dx.doi.org/10.21037/tau-21-400).

\section{Methods}

\section{Public databases}

Four available BCa cohorts, namely, GSE13507, TCGABLCA (23), E-MTAB-4321, and E-MTAB-1803 databases, were obtained from the Gene Expression Omnibus (GEO, http://www.ncbi.nlm.nih.gov/geo/), The Cancer Genome Atlas (TCGA, https://tcga-data.nci.nih.gov/), and the ArrayExpress database (https://www.ebi.ac.uk/ arrayexpress/). The HTSeq-counts of RNA-seq data and clinical data for $404 \mathrm{BCa}$ patients from the TCGABLCA project were downloaded and processed using the function in the R package, GDCRNAtools. The trimmed 
mean of the $M$ values (TMM) normalization of the count data was performed using edgeR. The processed gene expression profiles from GSE13507, E-MTAB-4321, and E-MTAB-1803 were utilized directly in the further analysis. All of the databases contained the sequencing results of the CYP19A1 expression of each patient. The BCa patients in each cohort were calibrated and divided into NMIBC and MIBC according to the 8th edition cancer staging system of the American Joint Committee on Cancer (24). Of a total of $165 \mathrm{BCa}$ patients (average age 65 years) from the GSE13507 BCa cohort, 104 patients (average age 64 years) were diagnosed as NMIBC, and 61 patients (average age 67 years) were diagnosed as MIBC. A total of $404 \mathrm{BCa}$ patients were diagnosed as MIBC (average age 68 years) from the TCGA database. A total of $460 \mathrm{BCa}$ patients were diagnosed as NMIBC (average age 69 years) from the E-MTAB-4321 BCa cohort. A total of 73 patients were diagnosed as MIBC (average age 67 years) from the E-MTAB-1803 BCa cohort. Detailed patient characteristics of each BCa cohort are shown in Tables S1-S7. The study was conducted in accordance with the Declaration of Helsinki (as revised in 2013) and no ethical approval was required because the data we used were obtained from public databases. Because of the retrospective nature of the research, the requirement for informed consent was waived.

\section{Gene set enrichment analysis}

To explore the potential biological alteration related to CYP19A1 in BCa, a differentially expressed analysis was first performed between the good- and poor-outcome subgroups based on the optimal cut-off value of CYP19A1 expression in the entire cohort or the NMIBC subgroup from GSE13507. We then obtained the gene list order via the decreasing levels of Log2FoldChange. The ordered gene list was further fitted in the GSEA analysis by the R package, 'clusterProfilter', using the annotation of 'hallmark gene sets.' A false discovery rate (FDR) $<0.25$ and adjusted $P$ value $<0.01$ were considered to be statistically significant.

\section{Statistical analysis}

A comparison of the CYP19A1 expression between the two subgroups in each cohort was analyzed using the Wilcoxon rank-sum test and is shown by Box Plots. The function, 'surv_cutpoint', in the R package, 'survminer' was implemented to discover the optimal cut-off value of CYP19A1 using the maximally selected rank statistics, which is an outcome-oriented method that provides a value of a cut point that corresponds to the most significant relation with the outcome (25). In addition, the cut-off values were used to determine the different CYP19A1 expression groups (high vs. low). The associations between the CYP19A1 expression level and the $\mathrm{BCa}$ patient characteristics in each cohort were analyzed using the Pearson's Chi-squared test. The OS and progression-free survival (PFS) were evaluated using the Kaplan-Meier method and the log-rank test based on the cut-off values using the survminer $\mathrm{R}$ package. All of the statistical tests were two-sided, and the p-value less than 0.05 was considered to be statistically significant.

\section{Results}

\section{Prognostic role of the CYP19A1 expression in the GSE13507 BCa cohort}

The prognostic role of the CYP19A1 expression in the GSE13507 cohort containing NMIBC and MIBC patients was first analyzed. Both patients were divided into high CYP19A1 expression group and low CYP19A1 expression group according to the cut-off value (Figure $\mathrm{S1A}$ ). As shown in Figure 1A, high expression of CYP19A1 was significantly associated with the poorer OS of BCa patients, which agreed with a previous study (19). The expression pattern of aromatase in BCa had been previously described $(19,20)$, and our analysis showed that there was no significant statistical difference in the CYP19A1 expression between the NMIBC and MIBC patients (Figure $1 B$ ). Still, the female patients showed higher CYP19A1 expression levels compared with the male patients (Figure 1C). In addition, the Kaplan-Meier analysis demonstrated that male patients with high CYP19A1 expression had a worse OS than those with low CYP19A1 expression (Figure 1D, Figure S1B), whereas female patients with high CYP19A1 had a better prognosis (Figure 1E, Figure S1C). Therefore, we hypothesized that different prognostic roles of CYP19A1 existed in the $\mathrm{BCa}$ patients under certain conditions. Our analysis further confirmed that CYP19A1 was more expressed in female NMIBC patients than in male NMIBC patients when we combined the gender and tumor stage (Figure $1 F$ ). However, there was no significant statistical difference in the CYP19A1 expression between male and female patients with MIBC (Figure 1G). Thus, we wondered that if a high CYP19A1 expression in the NMIBC patients shows a similar prognostic role to our analysis in Figure $1 A, D, E$, and the consistent results were observed (Figure 1H, Figure S1D). 
A

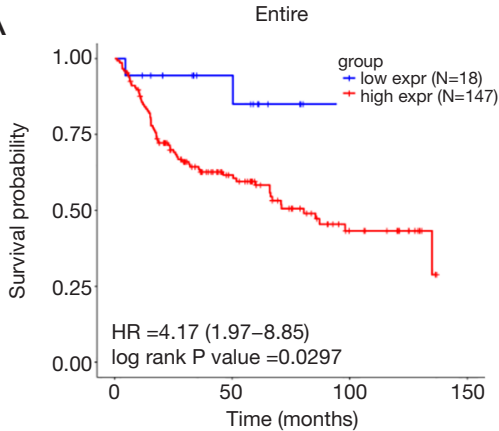

B

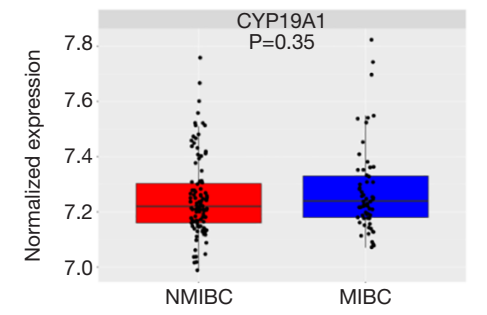

C

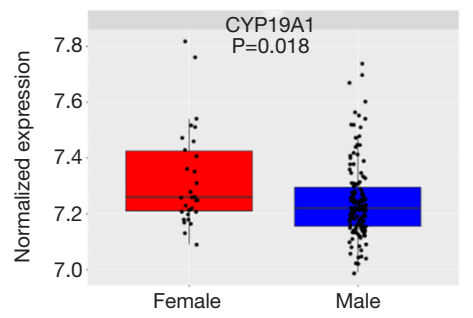

D

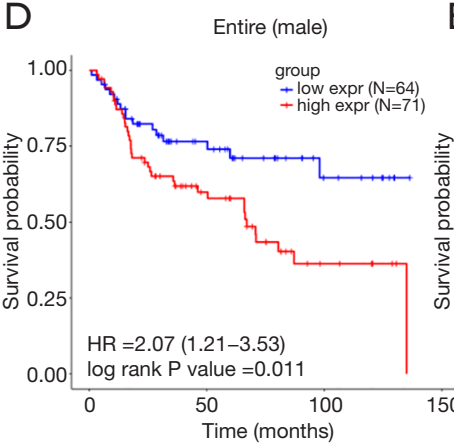

$\mathrm{H}$

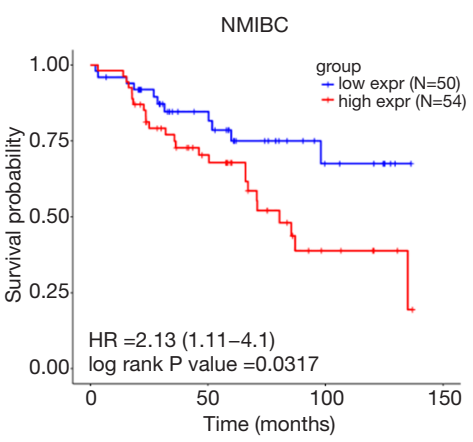

K

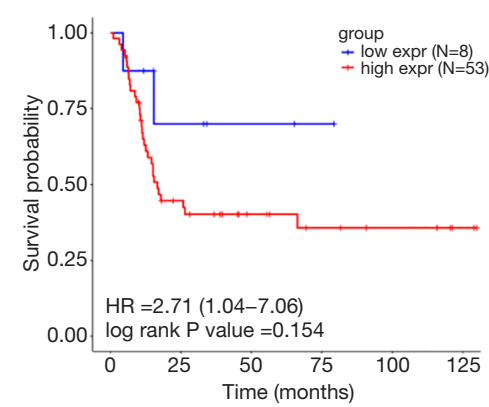

E
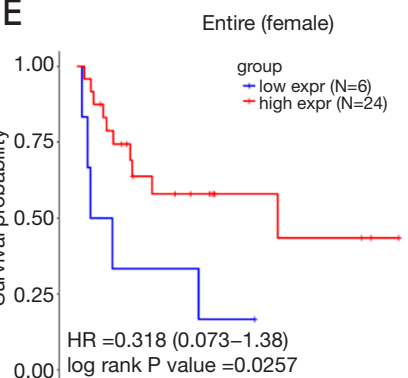

$0.00 . \log$ rank $P$ value $=0.0257$
$\mathrm{F}$

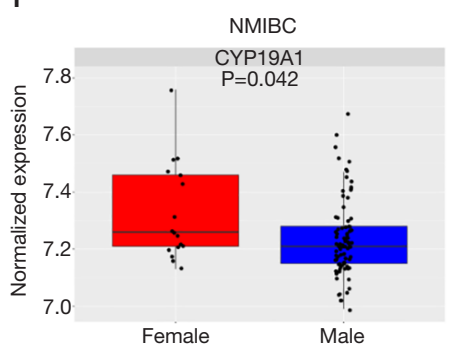

G

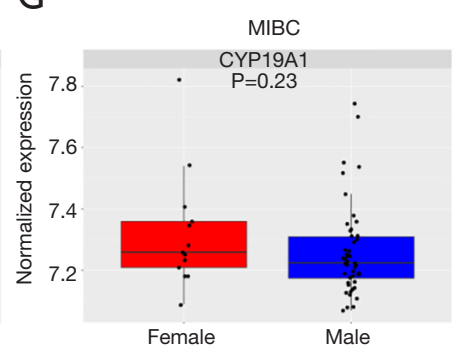

I

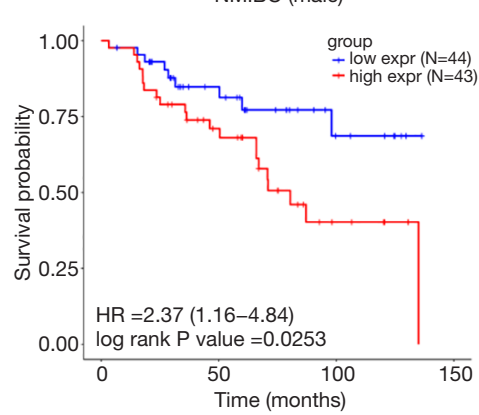

$\mathrm{L}$

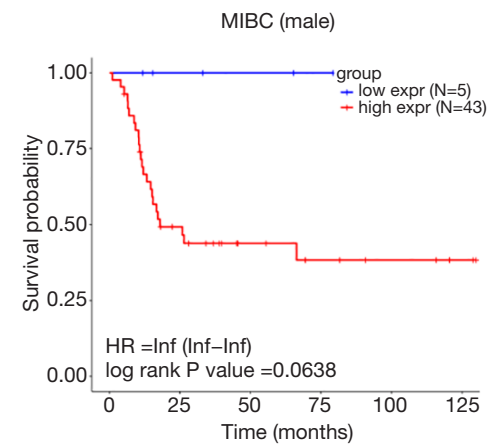

J

NMIBC (female)

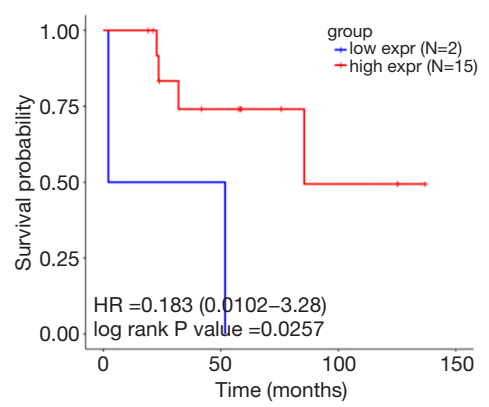

M

MIBC (female)

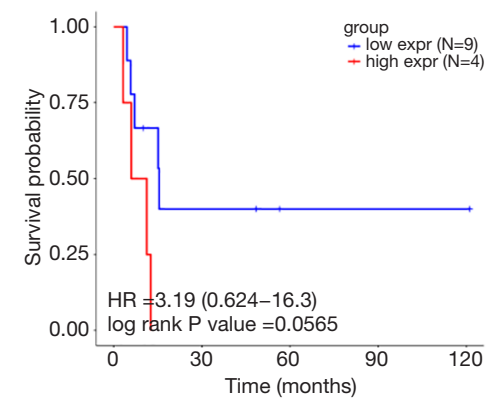

Figure 1 Prognostic roles of CYP19A1 in the GSE13507 BCa cohort. The overall survival analysis of CYP19A1 in the BCa patients (A). The expression level of CYP19A1 between the NMIBC patients and the MIBC patients (B), and between female and male BCa patients (C). The overall survival analysis of CYP19A1 in the male BCa patients (D) and the female BCa patients (E). The expression level of CYP19A1 between males and females in the NMIBC $(\mathrm{F})$ and MIBC $(\mathrm{G})$ patients. The overall survival analysis of CYP19A1 in all of the NMIBC patients (H), the male NMIBC patients (I), and the female NMIBC patients (J). The overall survival analysis of CYP19A1 in all of the MIBC patients $(\mathrm{K})$, the male MIBC patients $(\mathrm{L})$, and the female MIBC patients $(\mathrm{M})$. BCa, bladder cancer; NMIBC, non-muscle-invasive bladder cancer; MIBC, muscle-invasive bladder cancer. 
Table 1 The association between CYP19A1 expression level and the characteristics of male NMIBC patients in the GSE13507 BCa cohort

\begin{tabular}{ccccc}
\hline Variable & Number & $\begin{array}{c}\text { CYP19A1 } \\
\text { (high) }\end{array}$ & $\begin{array}{c}\text { CYP19A1 } \\
\text { (low) }\end{array}$ & P value \\
\hline Total & 87 & 43 & 44 & 1 \\
Age & 49 & 24 & 25 & \\
$\geq 65$ & 38 & 19 & 19 & 1 \\
$<65$ & 73 & 36 & 37 & \\
Grade & 14 & 7 & 7 & 1 \\
Low & & & & \\
High & 19 & 9 & 10 & \\
Stage & 68 & 34 & 34 & \\
Ta & & & & \\
T1 & & & & \\
\hline
\end{tabular}

NMIBC, non-muscle-invasive bladder cancer; $\mathrm{BCa}$, bladder cancer.

Table 2 The association between CYP19A1 expression level and the characteristics of female NMIBC patients in the GSE13507 BCa cohort

\begin{tabular}{ccccc}
\hline Variable & Number & $\begin{array}{c}\text { CYP19A1 } \\
\text { (high) }\end{array}$ & $\begin{array}{c}\text { CYP19A1 } \\
\text { (low) }\end{array}$ & P value \\
\hline Total & 17 & 15 & 2 & \\
Age & 10 & 8 & 2 & 0.4853 \\
$\geq 65$ & 7 & 7 & 0 & \\
$<65$ & 13 & 13 & 0 & 0.0677 \\
Grade & 4 & 2 & 2 & \\
Low & & & & 0.5147 \\
High & 5 & 4 & 1 & \\
Stage & 12 & 11 & 1 & \\
Ta & & & & \\
T1 & & & & \\
\hline
\end{tabular}

NMIBC, non-muscle-invasive bladder cancer; BCa, bladder cancer.

Surprisingly, the prognostic role of CYP19A1 in female NMIBC patients was contrary to that in male NMIBC patients (Figure 1I,17, Figure S1E,S1F). However, the roles of CYP19A1 in these two groups were not correlated with patient characteristics (Tables 1,2). Female NMIBC patients with high CYP19A1 expression were identified to have a better prognosis. Although CYP19A1 expression level had no impact on the OS when all of the MIBC patients were enrolled in our analysis (Figure $1 K$, Figure S1G), but we observed a trend that male and female MIBC patients with high CYP19A1 expression were more likely to have worse OS (Figure 1L,1M, Figure S1H,S1I). These findings suggested that the prognostic roles of CYP19A1 in BCa patients depended on gender difference and tumor stage.

\section{Prognostic role of CYP19A1 expression in the E-MTAB-4321 BCa cohort}

To achieve more evidence that CYP19A1 expression may predict the prognosis of NMIBC patients of different genders, the E-MTAB-4321 cohort was used. First, 460 NMIBC patients with detailed records of PFS in this cohort were divided into two groups: those with high CYP19A1 expression and those with low CYP19A1 expression according to the cut-off value (Figure S2A). The KaplanMeier analysis showed that patients with high CYP19A1 expression had a shorter PFS (Figure 2A). Unlike the results shown in Figure $1 F$, no difference in the CYP19A1 expression between the male and female patients was shown in this NMIBC cohort (Figure 2B). However, we found that high CYP19A1 expression in male NMIBC patients were significantly associated with a shorter PFS (Figure 2C, Figure S2B). This finding was consistent with the results of the GSE13507 cohort and suggested that male NMIBC patients with a low CYP19A1 expression may have a better prognosis. However, the expression level of CYP19A1 had no impact on the PFS of female NMIBC patients in this cohort (Figure 2D, Figure S2C). Taken together with the results of the GSE13507 cohort, it was concluded that the CYP19A1 expression may predict the different prognosis in male and female NMIBC patients.

\section{Prognostic role of CYP19A1 expression in the TCGA and E-MTAB-1803 BCa cobort}

To verify the prognostic role of CYP19A1 expression in the MIBC patients described above, we performed a further analysis in another two MIBC cohorts from the TCGA and the E-MTAB-1803 databases. The cut-off values were used to determine the high CYP19A1expression group and the low CYP19A1 expression group. MIBC patients with high CYP19A1 expression had a worse OS than those with low CYP19A1 expression (Figure 3A, Figure S3A). Also, no 
A

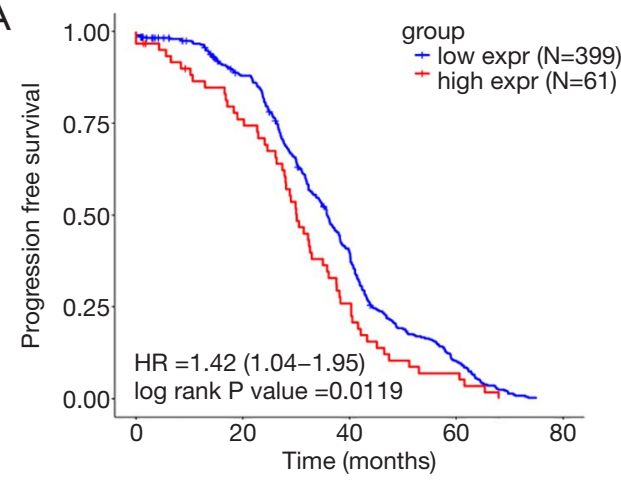

C

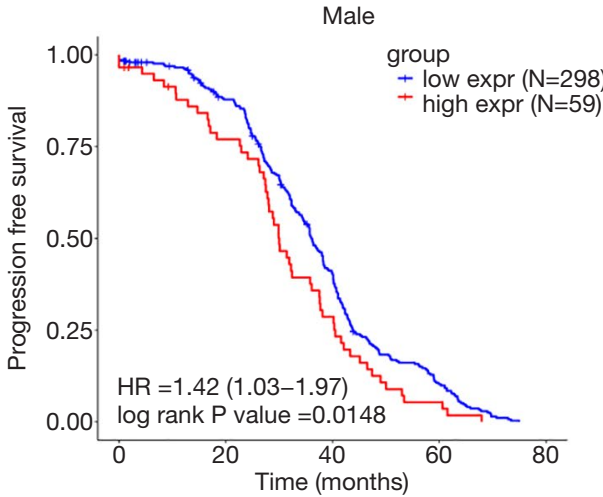

B
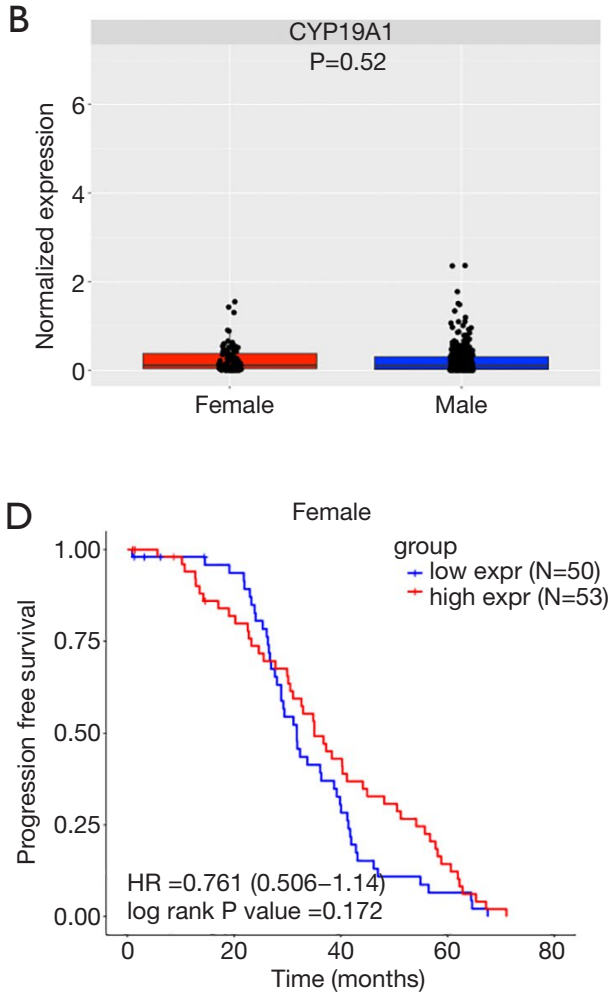

Figure 2 Prognostic roles of CYP19A1 in the E-MTBA-4321 BCa cohort. Progression-free survival analysis of CYP19A1 in the NMIBC BCa patients (A). The expression level of CYP19A1 between the male and female patients in the E-MTBA-4321 BCa cohort (B). Progression-free survival analysis of CYP19A1 in the male NMIBC patients (C) and the female NMIBC patients (D). BCa, bladder cancer; NMIBC, non-muscle-invasive bladder cancer.

difference in CYP19A1 expression between male and female patients in the MIBC patients was observed (Figure 3B). Additionally, male patients with different CYP19A1 expression in the TCGA cohort showed difference tendency but was not statistically significant (Figure 3C, Figure S3B), whereas those female patients with low CYP19A1 expression had a better prognosis (Figure 3D, Figure S3C). In order to verify the above result, similar analysis was performed in E-MTAB-1803. As shown in Fig 4A, MIBC patients with high CYP19A1 expression had a worse OS than those with low CYP19A1 expression (Figure 4A, Figure S3D). In line with the above result, there was no statistically significant difference between high and low expression of CYP19A1 in E-MTAB-1803 cohort (Figure 4B). Moreover, both male and female MIBC patients with high CYP19A1 expression had worse OS than those with low CYP19A1 expression in the E-MTAB-1803 cohort (Figure 4C,4D, Figure S3E,S3F). Taken together, these findings suggested that the high expression of CYP19A1 in MIBC patients may predict a poorer prognosis, either male or female.

\section{Functional enrichment analysis of the different expression levels of CYP19A1 in the NMIBC}

Given the different influence on the prognostic outcome of CYP19A1 in the NMIBC, we further explored the potential pathways that might possibly be influenced by CYP19A1 related to the different survival outcomes between males and females. We performed a GSEA in the NMIBC subgroup in the different genders in GSE13507, as mentioned above. The result of the significant gene set enrichment is shown in Tables S8,S9, and the enrichment map is shown in Figure 5. As shown in Figure $5 A, B$, cell-cycle-related pathway, 'HALLMARK_MYC_TARGETS_V1', 'HALLMARK E2 F_TARGETS' and 'HALLMARK_G2 M CHECKPOINT' (Figure 5C-5E) were suppressed in the female subgroup with high expression levels of CYP19A1 in the NMIBC patients. However, 'HALLMARK_MYC_ 
A

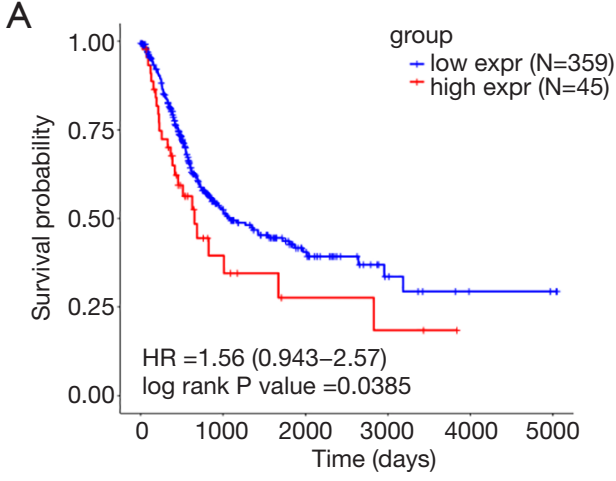

C

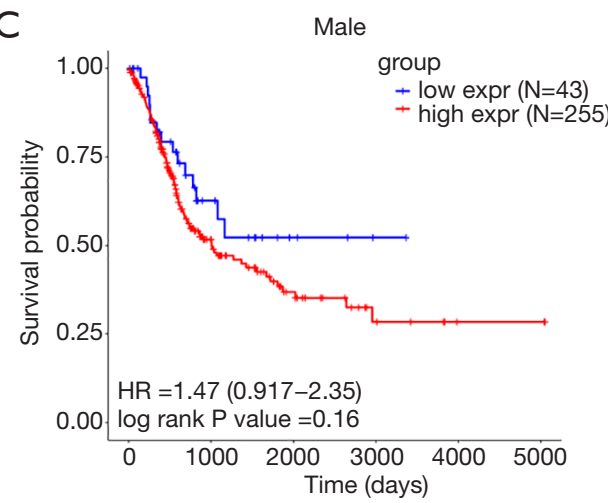

B
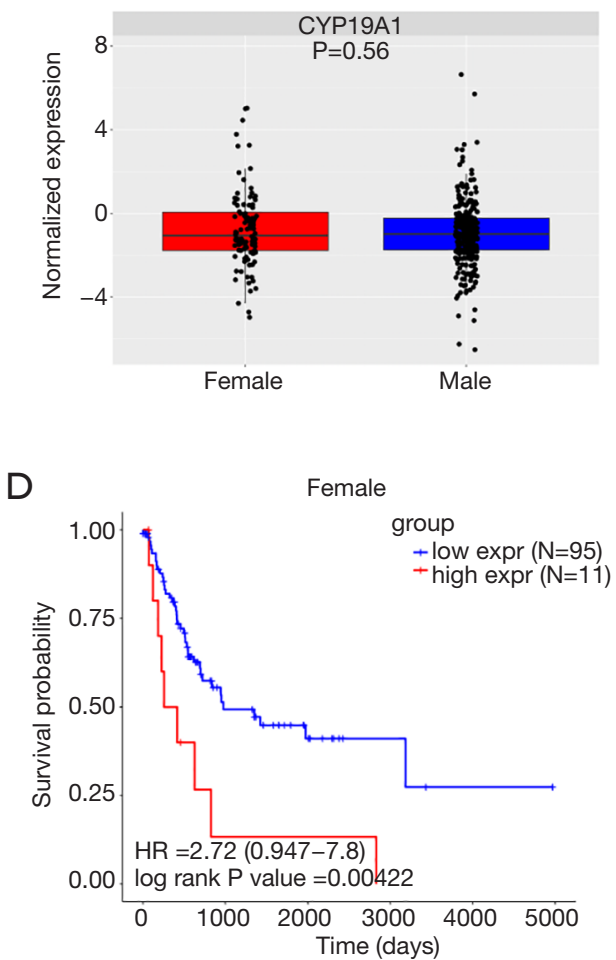

Figure 3 Prognostic roles of CYP19A1 in the TCGA BCa cohort. The overall survival analysis of CYP19A1 in the MIBC BCa patients (A). The expression level of CYP19A1 between the male and female patients in the TCGA BCa cohort (B). The overall survival analysis of CYP19A1 in the male MIBC patients (C) and the female MIBC patients (D). BCa, bladder cancer; MIBC, muscle-invasive bladder cancer.

TARGETS_V2', 'HALLMARK_G2M_CHECKPOINT' (Figure $5 F, 5 G$ ) were activated in the male subgroup with high expression levels of CYP19A1. This suggested that the activity of the cell cycle pathway, which is influenced by the expression of CYP19A1, may have contributed to the different survival outcomes in the NMIBC male and female patients.

\section{Discussion}

In this study, we investigated the association of the CYP19A1 expression level and patient prognosis in four public databases that contained clinical records and sequencing results. Although there were slight differences between the results of each BCa cohort, we were still able to observe a common trend that the prognostic roles of CYP19A1 in the BCa patients needed to be distinguished using a combined analysis of gender difference and tumor stage. Overall, patients with high CYP19A1 expression had poorer prognosis compared with those with low expression level in both $\mathrm{BCa}$ cohorts.
In male BCa patients, for either NMIBC or MIBC, a high CYP19A1 expression was significantly associated with shorter OS and PFS. However, a high expression of CYP19A1 in female NMIBC patients was identified to predict a longer OS, whereas its low expression predicted a better prognosis in female MIBC patients.

The common view is that males have a higher incidence and lower mortality of $\mathrm{BCa}$ compared with females. However, female $\mathrm{BCa}$ patients more frequently have advanced disease and poorer prognosis. Thus, it is necessary to identify novel biomarkers to distinguish the prognosis of male and female patients. Studies have demonstrated that the different behaviors between male and female BCa patients are independent of risk factors and clinicopathological features $(3,7)$. It follows that sex hormone have potential roles in the progression of $\mathrm{BCa}$. Overexpression of the androgen receptor (AR) can lead to the activation of $\mathrm{Wnt} /$ $\beta$-catenin signaling, which is significantly associated with $\mathrm{BCa}$ progression (26). Another study identified a novel ADAR2/ circFNTA/miR-370-3p/FNTA/KRAS axis driven by AR that 
A

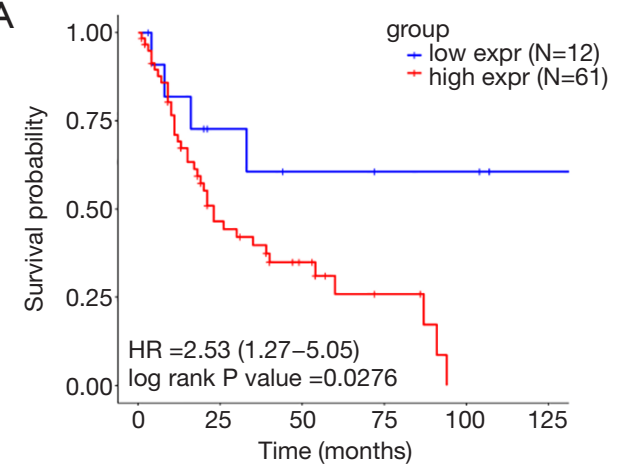

C

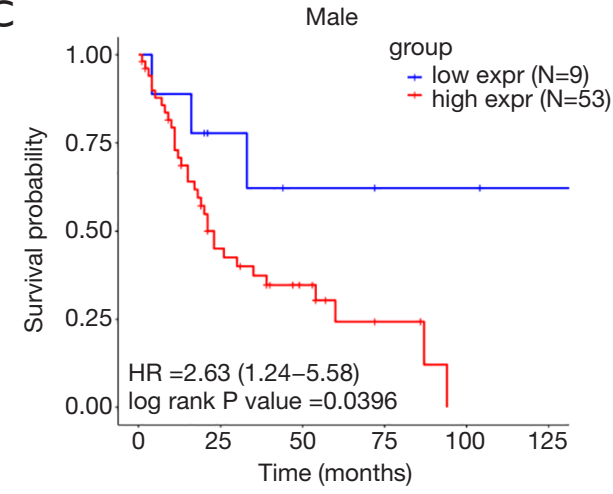

B
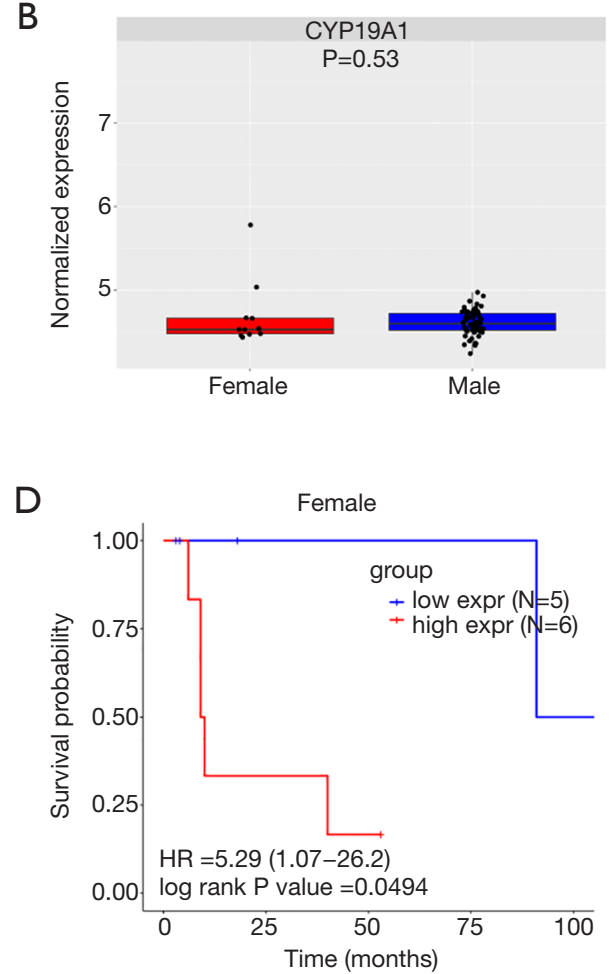

Figure 4 Prognostic roles of CYP19A1 in the E-MTAB-1803 BCa cohort. The overall survival analysis of CYP19A1 in the MIBC BCa patients (A). The expression level of CYP19A1 between the male and female patients in the E-MTAB-1803 BCa cohort (B). The overall survival analysis of CYP19A1 in the male MIBC patients (C) and the female MIBC patients (D). BCa, bladder cancer; MIBC, muscleinvasive bladder cancer.

may promote the invasion ability and the cisplatin chemoresistance of $\mathrm{BCa}$ (27). In addition, the overexpression of $\mathrm{AR}$ was found to induce the activation of a transcription factor, ELK1, and proto-oncogenes in BCa cells, promoting the proliferation of $\mathrm{BCa}$ (28). Additionally, the estrogen receptor (ER) was demonstrated to promote the progression of $\mathrm{BCa}$ by altering some signaling pathways, such as involving in the $\mathrm{T}$ cells/ER $\beta$ / MET axis (29), regulating the miR-92a/ DAB2IP signal (30), and increasing the expression of the estrogen-responsive gene GREB1 (31). However, a study demonstrated conflicting results in which the loss of AR or ER $\alpha$ was significantly associated with a high grade of $\mathrm{BCa}$ (32). The prognostic roles of sex hormones seem to be contrary to the results of the above studies. Additionally, another study suggested that ER, including ER $\alpha$ and ER $\beta$, cannot predict the prognosis of $\mathrm{BCa}$ patients, although their expression was higher in malignant tissue than in benign tissue (33). Also, patients with increased AR expression had the opposite recurrence-free survival rates between MIBC and NMIBC (34). Thus, it is difficult to evaluate the balance of $\mathrm{AR}$ and $\mathrm{ER}$ expression in $\mathrm{BCa}$ and their related functions.

Aromatase is typically expressed in a wide range of human tissues and responsible for estrogen synthesis by utilizing androgens $(9,22)$. A study suggested that the expression of aromatase can be upregulated by growth factors in adipose tissue. Based on this, increased ER production can facilitate growth factor synthesis by tumor cells (9). Therefore, the aberrant expression of aromatase will lead to an imbalance in AR and ER expression, which may have significant impacts on hormone-sensitive tumors. It has been reported that the majority of breast cancers express high concentrations of ER (35). As aromatase was abundantly expressed in adipose tissue adjacent to breast cancer tissue (13), the application of an aromatase inhibitor as an adjuvant therapy has been proven to have favorable clinical efficacy (36). In addition, prostate cancer is sensitive to AR and benefits from the reprograming of lipid metabolism. The role of CYP19A1 in prostate cancer was found to bind to the lipid metabolismrelated gene CTBP1 and result in its down-regulation. However, the metabolic syndrome of prostate cancer can 

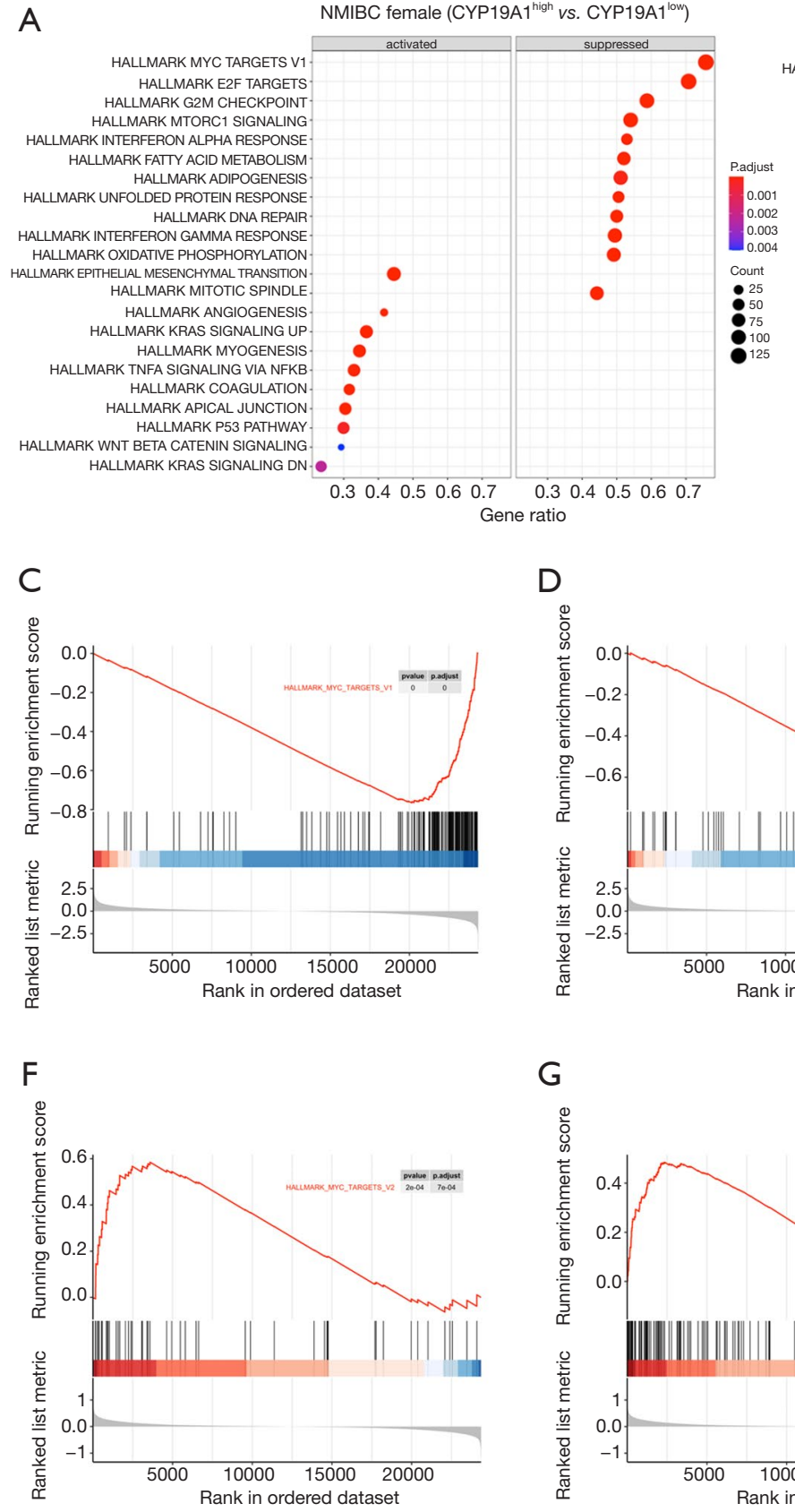

B

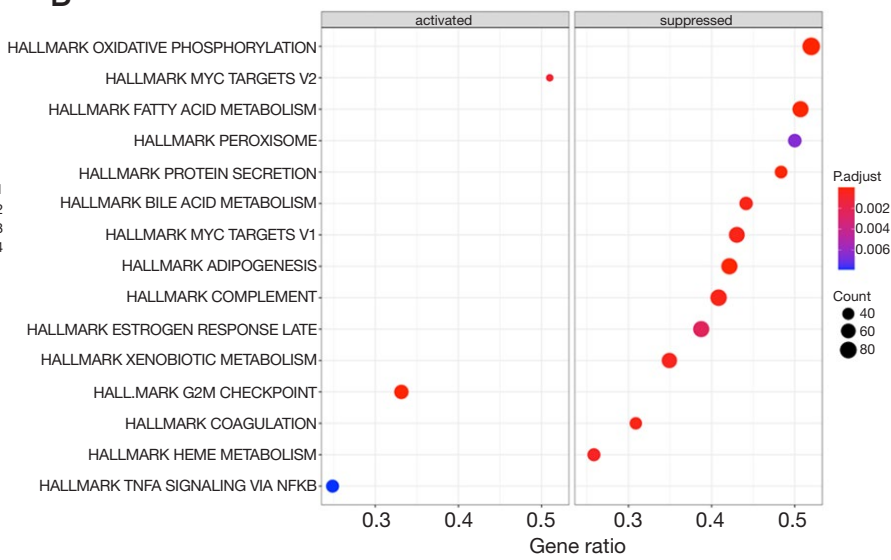

$\mathrm{E}$

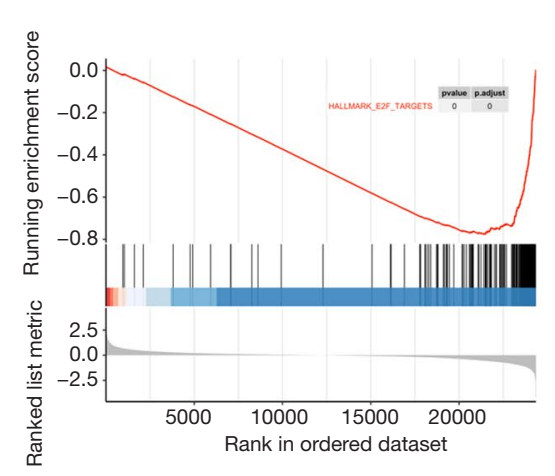

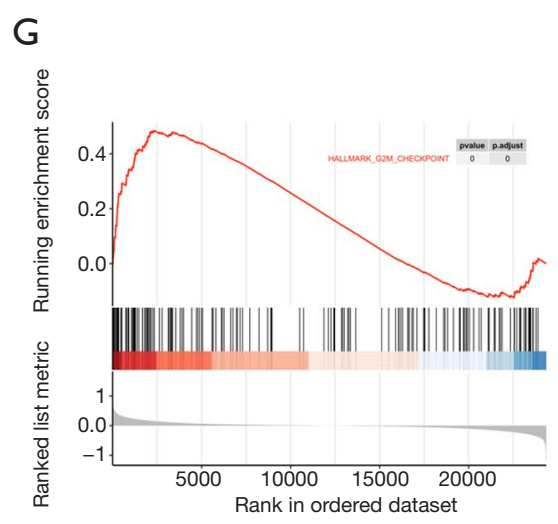

Figure 5 GSEA results of the different expression levels of CYP19A1 in the NMIBC subgroup. The most involved significant hallmarks pathways between CYP19A1 $1^{\text {high }}$ and CYP19A1 ${ }^{\text {low }}$ in the females with NMIBC subgroup from GSE13507 (A) and in the males with NMIBC subgroup from GSE13507 (B). The results of the cell-cycle-related pathways in the GSEA in the different expression levels of CYP19A1 in female patients with NMIBC (C-E) and in male patients with NMIBC (F-G). GSEA, Gene Set Enrichment Analyses; NMIBC, nonmuscle-invasive bladder cancer.

lead to an increased concentration of serum estradiol, which can release CTBP1 from the CYP19A1 promoter (14). Endogenous estrogen exposure is associated with increased endometrial cancer risk, and a study identified that the CYP19 haplotype has been frequently detected in invasive endometrial cancer tissue (15). A similar result was also 
observed in ovarian cancer (16). Taken together, these studies suggest that aromatase has the potential function of regulating sex hormone, which is related to the occurrence and development of tumors.

The G2/M checkpoint is essential for malignancy tumor cells to prevent mitotic catastrophe from creating excessive DNA damage, since the G1 checkpoint is deactivated in many cancers. Some research study on treating cancers by targeting the G2/M checkpoint have been performed $(37,38)$. Stine Hole found a new treatment target and blocked cell cycle progression at the G2/M phase in aromatase inhibitor-resistant breast cancer cells. He found that the mechanism of aromatase inhibitor-resistance may relate to the G2/M transition in a cancer cell (39). In addition, it has been shown that the genes regulated by the oncoprotein MYC were related to CYP19A1. MYC increases the anabolic metabolism of major nutrients to sustain the biosynthetic needs of cancer cells. Increased MYC expression contributes to tumorigenesis in many cancers and is associated with endocrine therapy resistance in breast cancer $(40,41)$. By combining with the results shown above, CYP19A1 may contribute to a different prognosis via the cell-cycle pathway, i.e., the G2/M checkpoint, MYC, and E2F in different genders in NMIBC. However, more evidence is required to demonstrate the mechanism of how the cell-cycle pathway contributes to the outcome of $\mathrm{BCa}$ in sex differences under the regulation of CYP19A1. Most of the studies described above focused on the roles of $\mathrm{AR}$ and $\mathrm{ER}$ in $\mathrm{BCa}$, but the controversial results require further investigation. However, increased aromatase expression has been demonstrated to be significantly associated with poorer OS (19). Our bioinformatics analysis of the four available public BCa cohorts also yielded similar results. In addition, they suggested no significant statistical difference in the expression of aromatase between males and females, which was consistent with our recent work $(19,21)$. But we noticed that these conclusions might have been influenced by the insufficient number of samples. Additionally, aromatase has been found to show differential concentrations in the plasma of NMIBC and MIBC patients (20). Therefore, a distinction analysis of the prognostic roles of aromatase in male and female patients with NMIBC or MIBC needs to be performed. Remarkably, the results of the GSE13507 cohort demonstrated that female patients had a higher CYP19A1 expression than male patients, and this differential expression was mainly reflected in the NMIBC patients. Based on this, female NIMBC patients with high CYP19A1 expression were identified to have longer OS, which was different from our results of the other subgroups and even the other BCa cohorts. Additionally, the increased expression of CYP19A1 cannot prolong the PFS of female NIMBC patients, but it was not correlated with the worse outcome. Unlike previous studies (19-21), the opposite prognostic role of aromatase in female NMIBC patients was first discovered. In addition, we confirmed that this role is not associated with the characteristics of patients, although it displayed differences in NMIBC and MIBC. These findings repeatedly indicated that sex hormones affect the unique behaviors of $\mathrm{BCa}$ with unknown mechanisms. Although the limited sample sizes in the subgroup of female NMIBC patients failed to draw a definitive conclusion, the specific molecular mechanisms of aromatase in $\mathrm{BCa}$ deserve future investigation.

\section{Conclusions}

In our study, we demonstrated for the first time that CYP19A1 offers a better prognosis in BCa patients of different genders according to tumor stage. Female NMIBC patients with high CYP19A1 expression are more likely to have a better prognosis, whereas high CYP19A1 expression in female MIBC patients were significantly associated with a more unsatisfactory outcome. The different prognosis in female and male patients with NMIBC were related to the interaction of CYP19A1 and cell-cycle-related pathways. Female BCa patients with varying stages of tumors and CYP19A1 expression require more attention when an aromatase inhibitor is used. Our findings provide new insights into aromatase-mediated BCa therapy.

\section{Acknowledgments}

We appreciate TCGA and GEO project team. We thank LetPub (www.letpub.com) for its linguistic assistance during the preparation of this manuscript.

Funding: This work was supported by National Natural Science Foundation of China (82072813, 81600620), Natural Science Foundation of Guangdong Province (2020A1515010473, 2020A1515110792), China Postdoctoral Science Foundation (2020M682666), The Science and Technology Development Fund (FDCT) of Macau SAR (0031/2021/A) and Guangzhou Municipal Science and Technology Project (201803040001). 


\section{Footnote}

Reporting Checklist: The authors have completed the MDAR checklist. Available at https://dx.doi.org/10.21037/tau-21-400

Conflicts of Interest: All authors have completed the ICMJE uniform disclosure form (available at https://dx.doi. org/10.21037/tau-21-400). The authors have no conflicts of interest to declare.

Ethical Statement: The authors are accountable for all aspects of the work in ensuring that questions related to the accuracy or integrity of any part of the work are appropriately investigated and resolved. The study was conducted in accordance with the Declaration of Helsinki (as revised in 2013) and no ethical approval was required because the data we used were obtained from public databases. Because of the retrospective nature of the research, the requirement for informed consent was waived.

Open Access Statement: This is an Open Access article distributed in accordance with the Creative Commons Attribution-NonCommercial-NoDerivs 4.0 International License (CC BY-NC-ND 4.0), which permits the noncommercial replication and distribution of the article with the strict proviso that no changes or edits are made and the original work is properly cited (including links to both the formal publication through the relevant DOI and the license). See: https://creativecommons.org/licenses/by-nc-nd/4.0/.

\section{References}

1. Bray F, Ferlay J, Soerjomataram I, et al. Global cancer statistics 2018: GLOBOCAN estimates of incidence and mortality worldwide for 36 cancers in 185 countries. CA Cancer J Clin 2018;68:394-424.

2. Cumberbatch MGK, Jubber I, Black PC, et al. Epidemiology of Bladder Cancer: A Systematic Review and Contemporary Update of Risk Factors in 2018. Eur Urol 2018;74:784-95.

3. Dobruch J, Daneshmand S, Fisch M, et al. Gender and Bladder Cancer: A Collaborative Review of Etiology, Biology, and Outcomes. Eur Urol 2016;69:300-10.

4. Kamat AM, Hahn NM, Efstathiou JA, et al. Bladder cancer. Lancet 2016;388:2796-810.

5. Miller KD, Nogueira L, Mariotto AB, et al. Cancer treatment and survivorship statistics, 2019. CA Cancer J Clin 2019;69:363-85.
6. Soave A, Dahlem R, Hansen J, et al. Gender-specific outcomes of bladder cancer patients: a stage-specific analysis in a contemporary, homogenous radical cystectomy cohort. Eur J Surg Oncol 2015;41:368-77.

7. Radkiewicz C, Edgren G, Johansson ALV, et al. Sex Differences in Urothelial Bladder Cancer Survival. Clin Genitourin Cancer 2020;18:26-34.e6.

8. Scosyrev E, Noyes K, Feng C, et al. Sex and racial differences in bladder cancer presentation and mortality in the US. Cancer 2009;115:68-74.

9. Simpson ER, Mahendroo MS, Means GD, et al. Aromatase cytochrome P450, the enzyme responsible for estrogen biosynthesis. Endocr Rev 1994;15:342-55.

10. Grodin JM, Siiteri PK, MacDonald PC. Source of estrogen production in postmenopausal women. J Clin Endocrinol Metab 1973;36:207-14.

11. Bulun SE, Chen D, Moy I, et al. Aromatase, breast cancer and obesity: a complex interaction. Trends Endocrinol Metab 2012;23:83-9.

12. Ohlsson C, Hammarstedt A, Vandenput L, et al. Increased adipose tissue aromatase activity improves insulin sensitivity and reduces adipose tissue inflammation in male mice. Am J Physiol Endocrinol Metab 2017;313:E450-62.

13. O'Neill JS, Miller WR. Aromatase activity in breast adipose tissue from women with benign and malignant breast diseases. Br J Cancer 1987;56:601-4.

14. Massillo C, Dalton GN, Porretti J, et al. CTBP1/ CYP19A1/estradiol axis together with adipose tissue impacts over prostate cancer growth associated to metabolic syndrome. Int J Cancer 2019;144:1115-27.

15. Paynter RA, Hankinson SE, Colditz GA, et al. CYP19 (aromatase) haplotypes and endometrial cancer risk. Int J Cancer 2005;116:267-74.

16. Cunat S, Rabenoelina F, Daurès JP, et al. Aromatase expression in ovarian epithelial cancers. J Steroid Biochem Mol Biol 2005;93:15-24.

17. Skjefstad K, Grindstad T, Khanehkenari MR, et al. Prognostic relevance of estrogen receptor $\alpha, \beta$ and aromatase expression in non-small cell lung cancer. Steroids 2016;113:5-13.

18. Sasano H, Sato S, Ito K, et al. Effects of aromatase inhibitors on the pathobiology of the human breast, endometrial and ovarian carcinoma. Endocr Relat Cancer 1999;6:197-204.

19. Nguyen DP, O'Malley $\mathrm{P}, \mathrm{Al}$ Hussein $\mathrm{Al}$ Awamlh B, et al. Association of Aromatase With Bladder Cancer Stage and Long-Term Survival: New Insights Into the Hormonal Paradigm in Bladder Cancer. Clin Genitourin Cancer 
2017;15:256-262.e1.

20. Guszcz T, Szymańska B, Kozlowski R, et al. Plasma aromatase as a sensitive and selective potential biomarker of bladder cancer and its role in tumorigenesis. Oncol Lett 2020;19:562-8.

21. Wu S, Ye J, Wang Z, et al. Expression of aromatase in tumor related stroma is associated with human bladder cancer progression. Cancer Biol Ther 2018;19:175-80.

22. Ghosh D, Griswold J, Erman M, et al. Structural basis for androgen specificity and oestrogen synthesis in human aromatase. Nature 2009;457:219-23.

23. Robertson AG, Kim J, Al-Ahmadie H, et al. Comprehensive Molecular Characterization of MuscleInvasive Bladder Cancer. Cell 2018;174:1033.

24. Amin MB, Greene FL, Edge SB, et al. The Eighth Edition AJCC Cancer Staging Manual: Continuing to build a bridge from a population-based to a more "personalized" approach to cancer staging. CA Cancer J Clin 2017;67:93-9.

25. Zeng D, Li M, Zhou R, et al. Tumor Microenvironment Characterization in Gastric Cancer Identifies Prognostic and Immunotherapeutically Relevant Gene Signatures. Cancer Immunol Res 2019; 7:737-50.

26. Li Y, Zheng Y, Izumi K, et al. Androgen activates $\beta$-catenin signaling in bladder cancer cells. Endocr Relat Cancer 2013;20:293-304.

27. Chen J, Sun Y, Ou Z, et al. Androgen receptor-regulated circFNTA activates KRAS signaling to promote bladder cancer invasion. EMBO Rep 2020;21:e48467.

28. Inoue S, Ide H, Mizushima T, et al. ELK1 promotes urothelial tumorigenesis in the presence of an activated androgen receptor. Am J Cancer Res 2018;8:2325-36.

29. Tao L, Qiu J, Slavin S, et al. Recruited T cells promote the bladder cancer metastasis via up-regulation of the estrogen receptor $\beta / \mathrm{IL}-1 / \mathrm{c}-\mathrm{MET}$ signals. Cancer Lett 2018;430:215-23.

30. Ou Z, Wang Y, Chen J, et al. Estrogen receptor $\beta$ promotes bladder cancer growth and invasion via alteration of miR-92a/DAB2IP signals. Exp Mol Med 2018;50:1-11.

31. Ding M, Liu Y, Li J, et al. Oestrogen promotes tumorigenesis of bladder cancer by inducing the enhancer RNA-eGREB1. J Cell Mol Med 2018;22:5919-27.

32. Miyamoto H, Yao JL, Chaux A, et al. Expression of androgen and oestrogen receptors and its prognostic significance in urothelial neoplasm of the urinary bladder. BJU Int 2012;109:1716-26.

33. Basakci A, Kirkali Z, Tuzel E, et al. Prognostic significance of estrogen receptor expression in superficial transitional cell carcinoma of the urinary bladder. Eur Urol 2002;41:342-5.

34. Yasui M, Kawahara T, Izumi K, et al. Androgen receptor mRNA expression is a predictor for recurrence-free survival in non-muscle invasive bladder cancer. BMC Cancer 2019;19:331.

35. Clark GM, Osborne CK, McGuire WL. Correlations between estrogen receptor, progesterone receptor, and patient characteristics in human breast cancer. J Clin Oncol 1984;2:1102-9.

36. Brueggemeier RW, Hackett JC, Diaz-Cruz ES. Aromatase inhibitors in the treatment of breast cancer. Endocr Rev 2005;26:331-45.

37. Chen T, Stephens PA, Middleton FK, et al. Targeting the $\mathrm{S}$ and G2 checkpoint to treat cancer. Drug Discov Today 2012;17:194-202.

38. Bucher N, Britten CD. G2 checkpoint abrogation and checkpoint kinase-1 targeting in the treatment of cancer. Br J Cancer 2008;98:523-8.

39. Hole S, Pedersen AM, Lykkesfeldt AE, et al. Aurora kinase $\mathrm{A}$ and $\mathrm{B}$ as new treatment targets in aromatase inhibitorresistant breast cancer cells. Breast Cancer Res Treat 2015;149:715-26.

40. Dejure FR, Eilers M. MYC and tumor metabolism: chicken and egg. EMBO J 2017;36:3409-20.

41. McNeil CM, Sergio CM, Anderson LR, et al. c-Myc overexpression and endocrine resistance in breast cancer. J Steroid Biochem Mol Biol 2006;102:147-55.
Cite this article as: Cai S, Feng Y, Ye J, Deng Y, Cai Z, Zhu X, Liu R, Zhang Y, Zou Z, Tang Z, Han Z, Hon CT, Zhong $\mathrm{W}, \mathrm{He} \mathrm{H}$. The prognostic roles of CYP19A1 expression in bladder cancer patients of different genders. Transl Androl Urol 2021;10(9):3579-3590. doi: 10.21037/tau-21-400 
Table S1 Patient characteristics of the GSE13507 BCa cohort

\begin{tabular}{|c|c|c|c|c|}
\hline Variable & Number & $\begin{array}{l}\text { CYP19A1 } \\
\text { (high) }\end{array}$ & $\begin{array}{l}\text { CYP19A1 } \\
\text { (low) }\end{array}$ & $P$ value \\
\hline Total & 165 & 147 & 18 & \\
\hline Age & & & & 0.4559 \\
\hline$\geq 65$ & 96 & 87 & 9 & \\
\hline$<65$ & 69 & 60 & 9 & \\
\hline Gender & & & & 0.1412 \\
\hline Male & 135 & 118 & 17 & \\
\hline Female & 30 & 29 & 1 & \\
\hline Grade & & & & 0.7771 \\
\hline Low & 105 & 93 & 12 & \\
\hline High & 60 & 54 & 6 & \\
\hline Stage & & & & 0.6632 \\
\hline $\mathrm{Ta}$ & 24 & 20 & 4 & \\
\hline $\mathrm{T} 1$ & 80 & 72 & 8 & \\
\hline T2 & 31 & 28 & 3 & \\
\hline T3 & 19 & 18 & 1 & \\
\hline $\mathrm{T} 4$ & 11 & 9 & 2 & \\
\hline
\end{tabular}

BCa, bladder cancer.

Table S2 Patient characteristics of the TCGA-BLCA BCa cohort

\begin{tabular}{|c|c|c|c|c|}
\hline Variable & Number & $\begin{array}{l}\text { CYP19A1 } \\
\text { (high) }\end{array}$ & $\begin{array}{l}\text { CYP19A1 } \\
\text { (low) }\end{array}$ & $P$ value \\
\hline Total & 404 & 45 & 359 & \\
\hline Age & & & & 0.1871 \\
\hline$\geq 68$ & 214 & 28 & 186 & \\
\hline$<68$ & 190 & 17 & 173 & \\
\hline Gender & & & & 0.1317 \\
\hline Male & 298 & 29 & 269 & \\
\hline Female & 106 & 16 & 90 & \\
\hline Stage & & & & 0.1402 \\
\hline $\mathrm{T} 2$ & 120 & 12 & 108 & \\
\hline T3 & 193 & 24 & 169 & \\
\hline $\mathrm{T} 4$ & 58 & 2 & 56 & \\
\hline Lymph node & & & & 0.7329 \\
\hline No & 234 & 27 & 207 & \\
\hline $\mathrm{N} 1$ & 46 & 6 & 40 & \\
\hline N2 & 75 & 6 & 69 & \\
\hline N3 & 8 & 1 & 7 & \\
\hline Metastasis & & & & 0.0684 \\
\hline Mo & 194 & 19 & 175 & \\
\hline M1 & 11 & 3 & 8 & \\
\hline Histologic subtype & & & & 0.3885 \\
\hline Non-papillary & 270 & 33 & 237 & \\
\hline Papillary & 129 & 12 & 117 & \\
\hline
\end{tabular}

$\mathrm{BCa}$, bladder cancer.

\begin{tabular}{|c|c|c|c|c|}
\hline Variable & Number & $\begin{array}{l}\text { CYP19A1 } \\
\text { (high) }\end{array}$ & $\begin{array}{l}\text { CYP19A1 } \\
\text { (low) }\end{array}$ & $P$ value \\
\hline Total & 460 & 61 & 399 & \\
\hline Age & & & & 0.6623 \\
\hline$\geq 65$ & 298 & 38 & 260 & \\
\hline$<65$ & 162 & 23 & 139 & \\
\hline Gender & & & & 0.6582 \\
\hline Male & 357 & 46 & 311 & \\
\hline Female & 103 & 15 & 88 & \\
\hline Grade & & & & 0.5160 \\
\hline Low & 277 & 35 & 242 & \\
\hline High & 176 & 26 & 150 & \\
\hline Stage & & & & 0.0283 \\
\hline Cis & 3 & 0 & 3 & \\
\hline $\mathrm{Ta}$ & 345 & 38 & 307 & \\
\hline T1 & 112 & 23 & 89 & \\
\hline
\end{tabular}

BCa, bladder cancer.

Table S4 Patient characteristics of the E-MTAB-1803 BCa cohort

\begin{tabular}{lcccc}
\hline Variable & Number & $\begin{array}{c}\text { CYP19A1 } \\
\text { (high) }\end{array}$ & $\begin{array}{c}\text { CYP19A1 } \\
\text { (low) }\end{array}$ & P value \\
\hline $\begin{array}{l}\text { Total } \\
\text { Age }\end{array}$ & 73 & 10 & 63 & \\
$\geq 65$ & 43 & 5 & 38 & 0.5379 \\
$<65$ & 30 & 5 & 25 & \\
Gender & & & & 0.5022 \\
Male & 62 & 8 & 54 & \\
Female & 11 & 2 & 9 & \\
Grade & & & & 0.4124
\end{tabular}

$\begin{array}{cccc}\text { G2 } & 4 & 0 & 4 \\ \text { G3 } & 69 & 10 & 59\end{array}$

Stage 0.1029

$\begin{array}{llll}\text { T2 } & 25 & 1 & 24\end{array}$

$\begin{array}{llll}\text { T3 } & 29 & 4 & 25\end{array}$

$\begin{array}{llll}\mathrm{T} 4 & 19 & 5 & 14\end{array}$

Lymph node $\quad 0.0280$

$\begin{array}{llll}\text { No } & 36 & 2 & 34 \\ \text { N1 } & 26 & 7 & 19\end{array}$

Metastasis $\quad 0.9433$

$\begin{array}{llll}\text { MO } & 59 & 8 & 51\end{array}$

$\begin{array}{llll}\text { M1 } & 14 & 2 & 12\end{array}$

Molecular subtype $\quad 0.9261$

\begin{tabular}{llll} 
Basal-like & 21 & 3 & 18 \\
Non-basal-like & 52 & 7 & 45 \\
\hline BCa, bladder cancer. & &
\end{tabular}


Table S5 Female patient characteristics of the TCGA-BLCA BCa cohort

\begin{tabular}{|c|c|c|c|}
\hline Factor & $\begin{array}{l}\text { CYP19A1 } \\
\text { (high) }\end{array}$ & $\begin{array}{l}\text { CYP19A1 } \\
\text { (low) }\end{array}$ & $P$ value \\
\hline Age & & & 0.6082 \\
\hline$\geq 68$ & 7 & 55 & \\
\hline$<68$ & 4 & 44 & \\
\hline Stage & & & 0.05587 \\
\hline T2 & 1 & 28 & \\
\hline T3 & 10 & 44 & \\
\hline $\mathrm{T} 4$ & 0 & 14 & \\
\hline Lymph node & & & 0.4992 \\
\hline No & 6 & 57 & \\
\hline N1-3 & 5 & 27 & \\
\hline Metastasis & & & 0.4048 \\
\hline Mo & 3 & 41 & \\
\hline M1 & 1 & 5 & \\
\hline MX & 7 & 51 & \\
\hline Histologic subtype & & & 0.2853 \\
\hline Non-papillary & 10 & 69 & \\
\hline Papillary & 1 & 25 & \\
\hline
\end{tabular}

$\mathrm{BCa}$, bladder cancer.

Table S6 Female patient characteristics of the E-MTAB-4321 BCa cohort

\begin{tabular}{cccc}
\hline Factor & CYP19A1 (high) & CYP19A1 (low) & P value \\
\hline Age & 33 & 31 & 0.978 \\
$\geq 65$ & 20 & 19 & \\
$<65$ & 34 & & 0.2526 \\
Grade & 18 & 26 & \\
Low & & 22 & \\
High & 0 & & \\
Stage & 41 & 39 & \\
cis & 12 & 10 & \\
Ta & & & \\
T1 & & & \\
\hline
\end{tabular}

$\mathrm{BCa}$, bladder cancer.
Table S7 Female patient characteristics of the E-MTAB-1803 BCa cohort

\begin{tabular}{|c|c|c|c|}
\hline Factor & $\begin{array}{l}\text { CYP19A1 } \\
\text { (high) }\end{array}$ & $\begin{array}{l}\text { CYP19A1 } \\
\text { (low) }\end{array}$ & $P$ value \\
\hline Age & & & 1 \\
\hline$\geq 67$ & 4 & 3 & \\
\hline$<67$ & 2 & 2 & \\
\hline Stage & & & 0.1775 \\
\hline T2 & 0 & 2 & \\
\hline T3 & 2 & 2 & \\
\hline $\mathrm{T} 4$ & 4 & 1 & \\
\hline Grade & & & 0.4545 \\
\hline G2 & 0 & 1 & \\
\hline G3 & 6 & 4 & \\
\hline Lymph node & & & 1 \\
\hline Negative & 3 & 3 & \\
\hline Positive & 3 & 2 & \\
\hline Distant metastasis & & & 1 \\
\hline Negative & 4 & 4 & \\
\hline Positive & 2 & 1 & \\
\hline
\end{tabular}

$\mathrm{BCa}$, bladder cancer. 
A
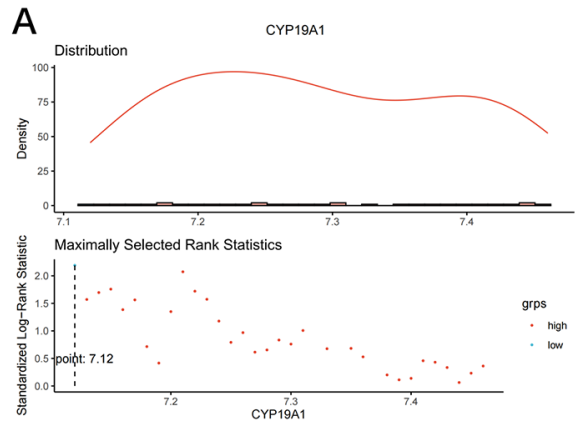

C
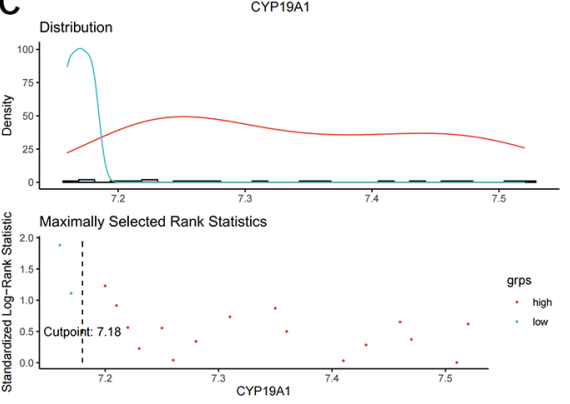

E
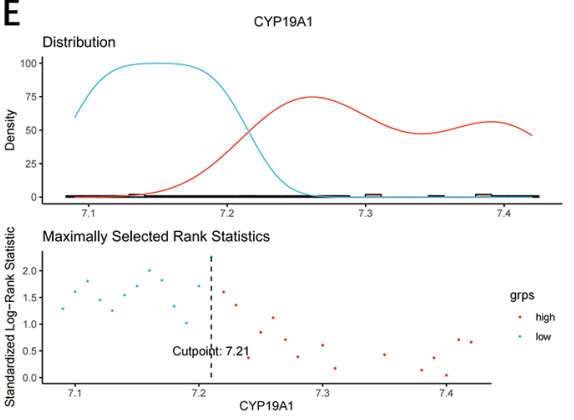

G
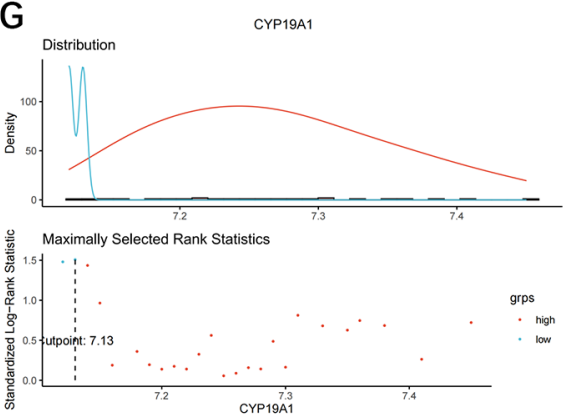

B
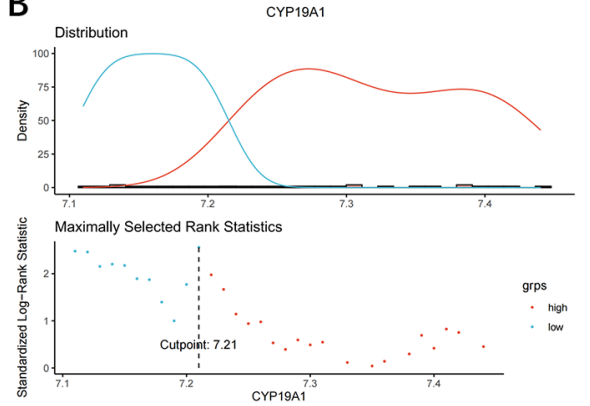

D CYP19A1
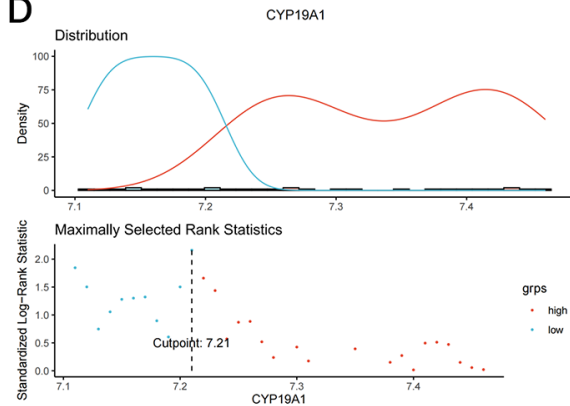

F
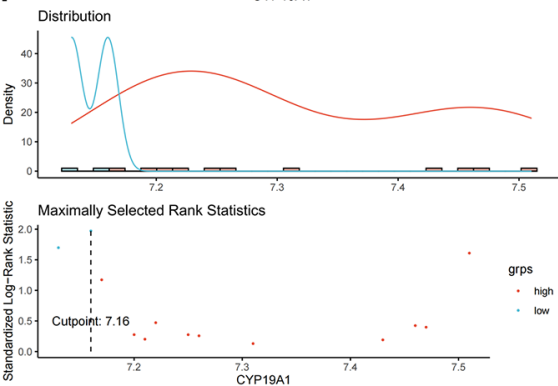

$\mathrm{H}$ CYP19A
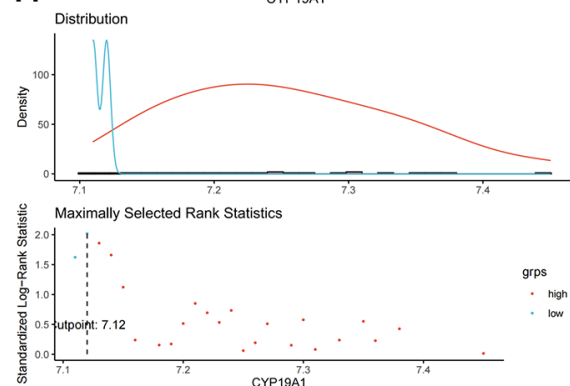

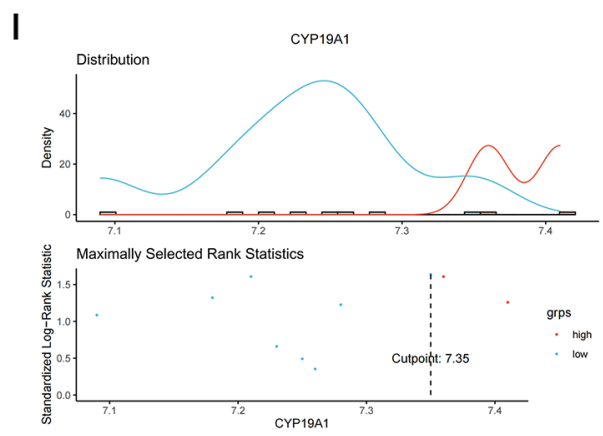

Figure S1 The cut-off value of CYP19A1 in the GSE13507 BCa cohort. (A) The cut-off value of CYP19A1 of entire patients in the GSE13507. (B) The cut-off value of CYP19A1 of all of the male patients in the GSE13507. (C) The cut-off value of CYP19A1 of all of the female patients in the GSE13507. (D) The cut-off value of CYP19A1 of NMIBC patients in the GSE13507. (E) The cut-off value of CYP19A1 of NMIBC male patients in the GSE13507. (F) The cut-off value of CYP19A1 of NMIBC female patients in the GSE13507. (G) The cut-off value of CYP19A1 of MIBC patients in the GSE13507. (H) The cut-off value of CYP19A1 of MIBC male patients in the GSE13507. (I) The cut-off value of CYP19A1 of MIBC female patients in the GSE13507. BCa, bladder cancer; NMIBC, non-muscleinvasive bladder cancer; MIBC, muscle-invasive bladder cancer. 

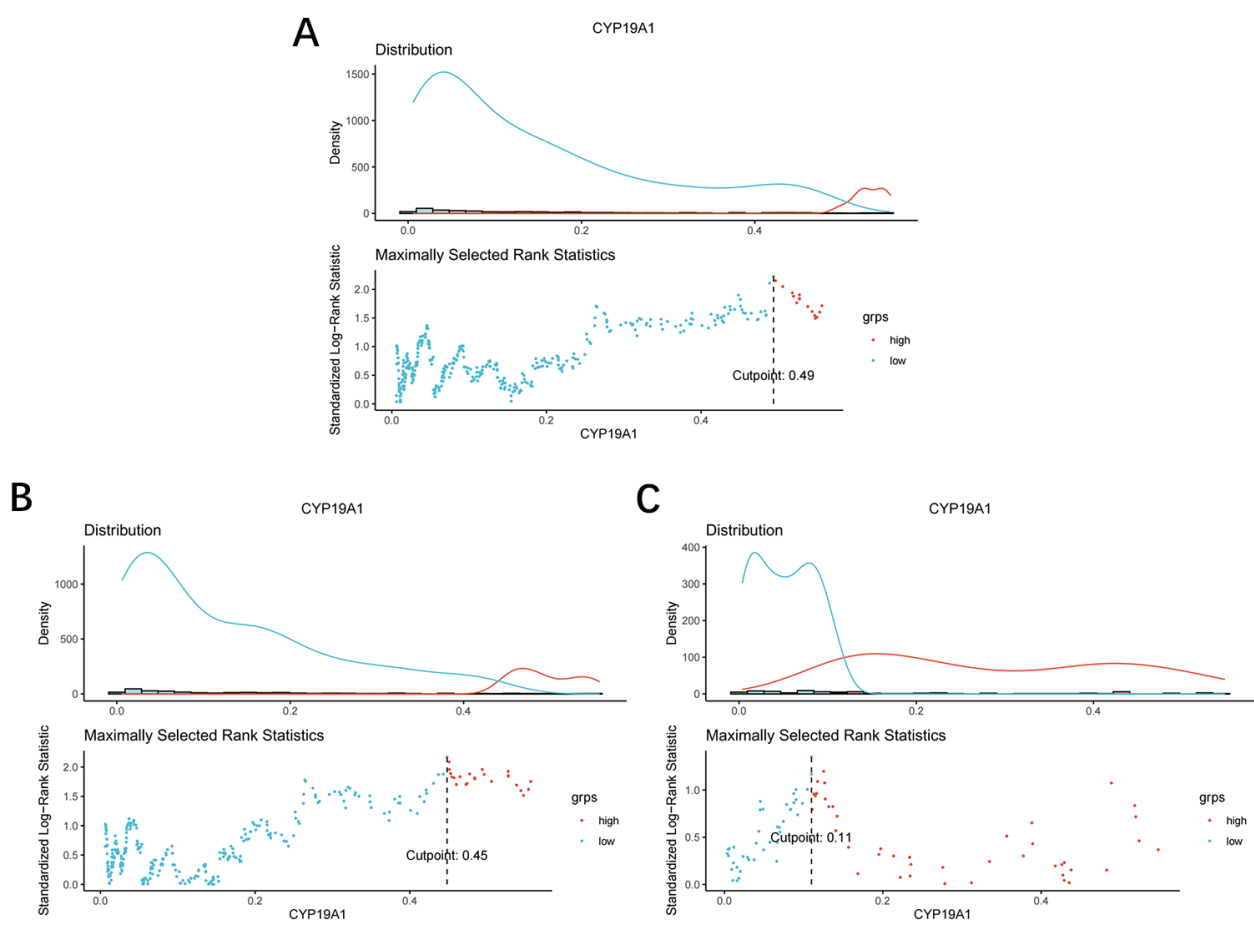

Figure S2 The cut-off value of CYP19A1 in the E-MTAB-4321 BCa cohort. (A) The cut-off value of CYP19A1 of NMIBC patients in the E-MTAB-4321. (B) The cut-off value of CYP19A1 of NMIBC male patients in the E-MTAB-4321. (C) The cut-off value of CYP19A1 of NMIBC female patients in the E-MTAB-4321. BCa, bladder cancer; NMIBC, non-muscle-invasive bladder cancer.

A
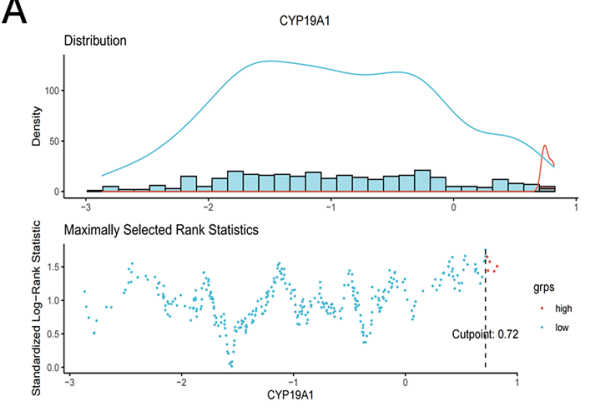

C

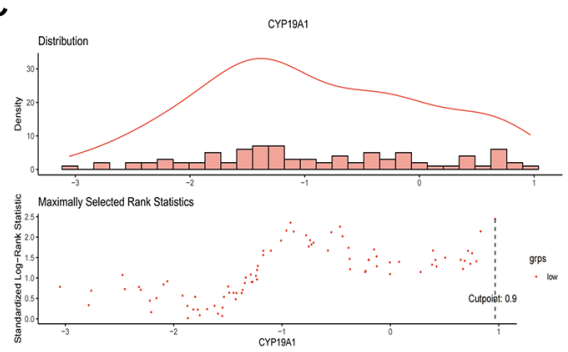

$\mathrm{E}$
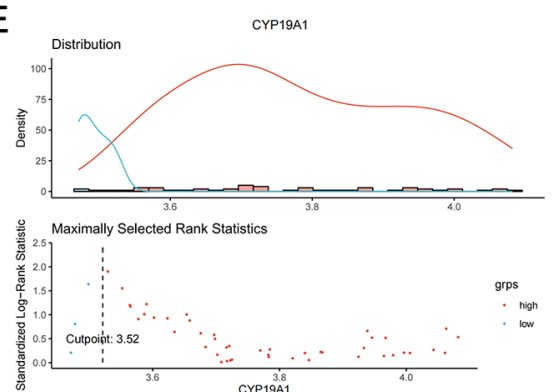

B
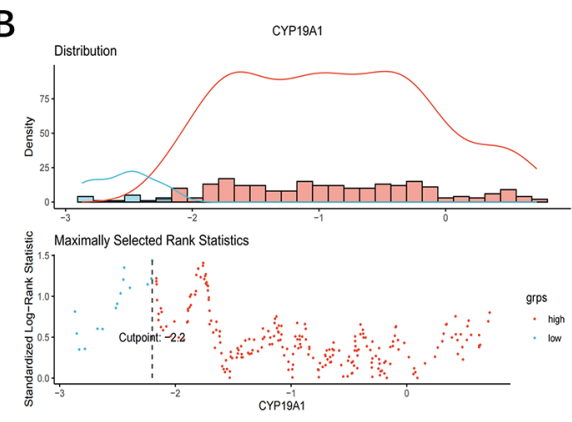

D

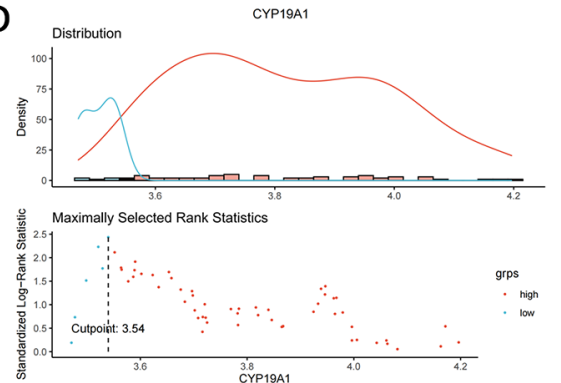

$\mathrm{F}$

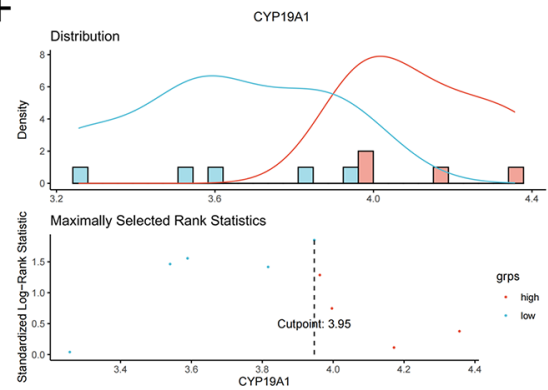

Figure S3 The cut-off value of CYP19A1 in the TCGA and E-MTAB-1803 BCa cohort. (A) The cut-off value of CYP19A1 of MIBC patients in the TCGA. (B) The cut-off value of CYP19A1 of MIBC male patients in the TCGA. (C) The cut-off value of CYP19A1 of MIBC female patients in the TCGA. (D) The cut-off value of CYP19A1 of MIBC patients in the E-MTAB-1803. (E) The cut-off value of CYP19A1 of MIBC male patients in the E-MTAB-1803. (F) The cut-off value of CYP19A1 of MIBC female patients in the E-MTAB-1803. $\mathrm{BCa}$, bladder cancer; MIBC, muscle-invasive bladder cancer. 
Table S8 Gene set enrichment analysis of NMIBC-male group in GSE13507

\begin{tabular}{|c|c|c|c|c|c|c|c|c|}
\hline ID & Set size & Enrichment score & NES & $P$ value & P.adjust & Qvalues & Rank & Leading edge \\
\hline HALLMARK_OXIDATIVE_PHOSPHORYLATION & 177 & -0.559967187 & -1.961959774 & 5.91E-09 & $2.95 \mathrm{E}-07$ & 1.37E-07 & 3434 & tags $=52 \%$, list $=14 \%$, signal $=45 \%$ \\
\hline HALLMARK_FATTY_ACID_METABOLISM & 146 & -0.547701502 & -1.885365225 & $4.26 \mathrm{E}-07$ & 1.07E-05 & 4.94E-06 & 4096 & tags $=51 \%$, list $=17 \%$, signal $=42 \%$ \\
\hline HALLMARK_G2M_CHECKPOINT & 172 & 0.485096805 & 1.856657182 & $1.33 \mathrm{E}-06$ & 2.02E-05 & $9.34 \mathrm{E}-06$ & 2345 & tags $=33 \%$, list $=10 \%$, signal $=30 \%$ \\
\hline HALLMARK_ADIPOGENESIS & 178 & -0.507484437 & -1.777359344 & $1.61 \mathrm{E}-06$ & 2.02E-05 & $9.34 \mathrm{E}-06$ & 4122 & tags $=42 \%$, list $=17 \%$, signal $=35 \%$ \\
\hline HALLMARK_PROTEIN_SECRETION & 91 & -0.584347118 & -1.898191198 & 7.59E-06 & 7.59E-05 & 3.52E-05 & 3612 & tags $=48 \%$, list $=15 \%$, signal $=41 \%$ \\
\hline HALLMARK_COMPLEMENT & 191 & -0.473142627 & -1.67101166 & 4.00E-05 & 0.000333035 & 0.000154248 & 5020 & tags $=41 \%$, list $=21 \%$, signal $=33 \%$ \\
\hline HALLMARK_COAGULATION & 136 & -0.502209567 & -1.7131716 & 5.49E-05 & 0.000392143 & 0.000181624 & 4118 & tags $=31 \%$, list $=17 \%$, signal $=26 \%$ \\
\hline HALLMARK_MYC_TARGETS_V1 & 165 & -0.483133051 & -1.68229054 & 7.12E-05 & 0.00044514 & 0.00020617 & 4371 & tags $=43 \%$, list $=18 \%$, signal $=36 \%$ \\
\hline HALLMARK_XENOBIOTIC_METABOLISM & 189 & -0.458627496 & -1.616879141 & 0.000126426 & 0.000599783 & 0.000277794 & 4168 & tags $=35 \%$, list $=17 \%$, signal $=29 \%$ \\
\hline HALLMARK_BILE_ACID_METABOLISM & 111 & -0.51633476 & -1.717279723 & 0.000126476 & 0.000599783 & 0.000277794 & 4894 & tags $=44 \%$, list $=20 \%$, signal $=35 \%$ \\
\hline HALLMARK_HEME_METABOLISM & 178 & -0.461348091 & -1.615776326 & 0.000131952 & 0.000599783 & 0.000277794 & 2853 & tags $=26 \%$, list $=12 \%$, signal $=23 \%$ \\
\hline HALLMARK_MYC_TARGETS_V2 & 51 & 0.584960812 & 1.894207379 & 0.000165795 & 0.000690814 & 0.000319956 & 3581 & tags $=51 \%$, list $=15 \%$, signal $=44 \%$ \\
\hline HALLMARK_ESTROGEN_RESPONSE_LATE & 191 & -0.441418566 & -1.558970865 & 0.000532383 & 0.002047628 & 0.000948375 & 3770 & tags $=39 \%$, list $=15 \%$, signal $=33 \%$ \\
\hline HALLMARK_TNFA_SIGNALING_VIA_NFKB & 185 & 0.369851266 & 1.435026511 & 0.002667351 & 0.009526254 & 0.00441216 & 2388 & tags $=25 \%$, list $=10 \%$, signal $=23 \%$ \\
\hline HALLMARK_PEROXISOME & 100 & -0.474382794 & -1.559911738 & 0.003178412 & 0.010594707 & 0.004907022 & 4978 & tags $=50 \%$, list $=20 \%$, signal $=40 \%$ \\
\hline HALLMARK_GLYCOLYSIS & 187 & -0.397701058 & -1.399293535 & 0.005473292 & 0.017104039 & 0.007921871 & 3263 & tags $=33 \%$, list $=13 \%$, signal $=28 \%$ \\
\hline HALLMARK_INTERFERON_GAMMA_RESPONSE & 188 & -0.399600864 & -1.407577725 & 0.006384718 & 0.018778583 & 0.008697449 & 4619 & tags $=39 \%$, list $=19 \%$, signal $=32 \%$ \\
\hline HALLMARK_ESTROGEN_RESPONSE_EARLY & 179 & -0.40732484 & -1.428327744 & 0.00728637 & 0.020071189 & 0.00929613 & 4616 & tags $=42 \%$, list $=19 \%$, signal $=35 \%$ \\
\hline HALLMARK_KRAS_SIGNALING_UP & 186 & -0.392343387 & -1.380691013 & 0.007838121 & 0.020071189 & 0.00929613 & 1803 & tags $=21 \%$, list $=7 \%$, signal $=20 \%$ \\
\hline HALLMARK_DNA_REPAIR & 134 & -0.425042603 & -1.445729777 & 0.008028476 & 0.020071189 & 0.00929613 & 5410 & tags $=44 \%$, list $=22 \%$, signal $=34 \%$ \\
\hline HALLMARK_APOPTOSIS & 157 & -0.407543588 & -1.412490092 & 0.008841272 & 0.021050647 & 0.009749774 & 4663 & tags $=45 \%$, list $=19 \%$, signal $=37 \%$ \\
\hline HALLMARK_P53_PATHWAY & 177 & -0.38845323 & -1.361025485 & 0.010492025 & 0.023845512 & 0.011044237 & 4361 & tags $=38 \%$, list $=18 \%$, signal $=32 \%$ \\
\hline HALLMARK_INTERFERON_ALPHA_RESPONSE & 85 & -0.467358559 & -1.514063802 & 0.011266286 & 0.024491926 & 0.011343629 & 4619 & tags $=45 \%$, list $=19 \%$, signal $=36 \%$ \\
\hline HALLMARK_ANDROGEN_RESPONSE & 91 & -0.448259421 & -1.456124383 & 0.014145996 & 0.028326867 & 0.013119812 & 3878 & tags $=42 \%$, list $=16 \%$, signal $=35 \%$ \\
\hline HALLMARK_IL2_STAT5_SIGNALING & 180 & -0.39331732 & -1.379268346 & 0.014163433 & 0.028326867 & 0.013119812 & 4689 & tags $=36 \%$, list $=19 \%$, signal $=29 \%$ \\
\hline
\end{tabular}

NMIBC, non-muscle-invasive bladder cancer. 
Table S9 Gene set enrichment analysis of NMIBC-female group in GSE13507

\begin{tabular}{|c|c|c|c|c|c|c|c|c|}
\hline ID & Set size & Enrichment score & NES & $P$ value & P.adjust & Qvalues & Rank & Leading edge \\
\hline HALLMARK_E2F_TARGETS & 171 & -0.776088779 & -2.614570306 & $1.00 \mathrm{E}-10$ & $8.33 \mathrm{E}-10$ & $3.16 \mathrm{E}-10$ & 2960 & tags $=71 \%$, list $=12 \%$, signal $=63 \%$ \\
\hline HALLMARK_EPITHELIAL_MESENCHYMAL_TRANSITION & 191 & 0.565614922 & 2.330145827 & $1.00 \mathrm{E}-10$ & 8.33E-10 & $3.16 \mathrm{E}-10$ & 2805 & tags $=45 \%$, list $=12 \%$, signal $=40 \%$ \\
\hline HALLMARK_G2M_CHECKPOINT & 172 & -0.723904873 & -2.441124828 & $1.00 \mathrm{E}-10$ & 8.33E-10 & $3.16 \mathrm{E}-10$ & 3381 & tags $=59 \%$, list $=14 \%$, signal $=51 \%$ \\
\hline HALLMARK_MTORC1_SIGNALING & 187 & -0.609516014 & -2.067773306 & $1.00 \mathrm{E}-10$ & 8.33E-10 & 3.16E-10 & 4567 & tags $=54 \%$, list $=19 \%$, signal $=44 \%$ \\
\hline HALLMARK_MYC_TARGETS_V1 & 165 & -0.765531016 & -2.565767189 & $1.00 \mathrm{E}-10$ & 8.33E-10 & $3.16 \mathrm{E}-10$ & 4208 & tags $=76 \%$, list $=17 \%$, signal $=63 \%$ \\
\hline HALLMARK_OXIDATIVE_PHOSPHORYLATION & 177 & -0.608743158 & -2.057054864 & $1.00 \mathrm{E}-10$ & 8.33E-10 & $3.16 \mathrm{E}-10$ & 4022 & tags $=49 \%$, list $=17 \%$, signal $=41 \%$ \\
\hline HALLMARK_INTERFERON_GAMMA_RESPONSE & 188 & -0.578995427 & -1.967531255 & $3.61 \mathrm{E}-10$ & $2.58 \mathrm{E}-09$ & $9.78 \mathrm{E}-10$ & 4765 & tags $=49 \%$, list $=20 \%$, signal $=40 \%$ \\
\hline HALLMARK_DNA_REPAIR & 134 & -0.610795203 & -2.007550375 & $7.95 \mathrm{E}-10$ & 4.97E-09 & $1.88 \mathrm{E}-09$ & 4221 & tags $=50 \%$, list $=17 \%$, signal $=42 \%$ \\
\hline HALLMARK_INTERFERON_ALPHA_RESPONSE & 85 & -0.67044919 & -2.09354555 & 1.77E-09 & $9.82 \mathrm{E}-09$ & $3.72 \mathrm{E}-09$ & 3479 & tags $=53 \%$, list $=14 \%$, signal $=46 \%$ \\
\hline HALLMARK_UNFOLDED_PROTEIN_RESPONSE & 97 & -0.646215803 & -2.057706399 & $6.92 \mathrm{E}-09$ & $3.46 \mathrm{E}-08$ & $1.31 \mathrm{E}-08$ & 4577 & tags $=51 \%$, list $=19 \%$, signal $=41 \%$ \\
\hline HALLMARK_KRAS_SIGNALING_UP & 186 & 0.479822645 & 1.977011152 & $1.48 \mathrm{E}-08$ & $6.75 \mathrm{E}-08$ & $2.56 \mathrm{E}-08$ & 2989 & tags $=37 \%$, list $=12 \%$, signal $=32 \%$ \\
\hline HALLMARK_FATTY_ACID_METABOLISM & 146 & -0.573788024 & -1.901995754 & $2.77 \mathrm{E}-08$ & $1.15 \mathrm{E}-07$ & $4.38 \mathrm{E}-08$ & 4756 & tags $=52 \%$, list $=20 \%$, signal $=42 \%$ \\
\hline HALLMARK_COAGULATION & 136 & 0.497030428 & 1.953699039 & $2.30 \mathrm{E}-07$ & $8.85 \mathrm{E}-07$ & 3.35E-07 & 3412 & tags $=32 \%$, list $=14 \%$, signal $=27 \%$ \\
\hline HALLMARK_APICAL_JUNCTION & 187 & 0.44626909 & 1.836345257 & 4.63E-07 & $1.65 \mathrm{E}-06$ & $6.27 \mathrm{E}-07$ & 2869 & tags $=30 \%$, list $=12 \%$, signal $=27 \%$ \\
\hline HALLMARK_MYOGENESIS & 191 & 0.434338633 & 1.789331068 & 8.73E-07 & $2.91 \mathrm{E}-06$ & $1.10 \mathrm{E}-06$ & 3806 & tags $=35 \%$, list $=16 \%$, signal $=29 \%$ \\
\hline HALLMARK_MITOTIC_SPINDLE & 183 & -0.513752713 & -1.741476401 & $2.43 \mathrm{E}-06$ & $7.61 \mathrm{E}-06$ & $2.88 \mathrm{E}-06$ & 5544 & tags $=44 \%$, list $=23 \%$, signal $=34 \%$ \\
\hline HALLMARK_ANGIOGENESIS & 36 & 0.679887097 & 2.155090084 & $1.17 \mathrm{E}-05$ & $3.45 \mathrm{E}-05$ & $1.31 \mathrm{E}-05$ & 2150 & tags $=42 \%$, list $=9 \%$, signal $=38 \%$ \\
\hline HALLMARK_TNFA_SIGNALING_VIA_NFKB & 185 & 0.410930681 & 1.6868688897 & $1.81 \mathrm{E}-05$ & $5.02 \mathrm{E}-05$ & $1.90 \mathrm{E}-05$ & 3362 & tags $=33 \%$, list $=14 \%$, signal $=29 \%$ \\
\hline HALLMARK_ADIPOGENESIS & 178 & -0.488690704 & -1.649471403 & $6.18 \mathrm{E}-05$ & 0.000162683 & $6.16 \mathrm{E}-05$ & 5199 & tags $=51 \%$, list $=21 \%$, signal $=41 \%$ \\
\hline HALLMARK_PEROXISOME & 100 & -0.550591448 & -1.756063668 & $7.88 \mathrm{E}-05$ & 0.000196993 & $7.47 \mathrm{E}-05$ & 3909 & tags $=43 \%$, list $=16 \%$, signal $=36 \%$ \\
\hline HALLMARK_P53_PATHWAY & 177 & 0.393978342 & 1.609003638 & 0.00013862 & 0.000330048 & 0.000125071 & 3056 & tags $=30 \%$, list $=13 \%$, signal $=26 \%$ \\
\hline HALLMARK_MYC_TARGETS_V2 & 51 & -0.61939341 & -1.803546659 & 0.000195342 & 0.000443959 & 0.000168237 & 4452 & tags $=53 \%$, list $=18 \%$, signal $=43 \%$ \\
\hline HALLMARK_PROTEIN_SECRETION & 91 & -0.523409907 & -1.651679526 & 0.000811579 & 0.001764301 & 0.000668577 & 5044 & tags $=51 \%$, list $=21 \%$, signal $=40 \%$ \\
\hline HALLMARK_SPERMATOGENESIS & 122 & -0.474038378 & -1.534629411 & 0.001137864 & 0.002287275 & 0.000866757 & 4823 & tags $=27 \%$, list $=20 \%$, signal $=22 \%$ \\
\hline HALLMARK_KRAS_SIGNALING_DN & 179 & 0.35405565 & 1.454771637 & 0.001143638 & 0.002287275 & 0.000866757 & 3235 & tags $=23 \%$, list $=13 \%$, signal $=20 \%$ \\
\hline HALLMARK_CHOLESTEROL_HOMEOSTASIS & 68 & -0.548560807 & -1.668260357 & 0.001477391 & 0.002763106 & 0.001047072 & 2443 & tags $=47 \%$, list $=10 \%$, signal $=42 \%$ \\
\hline HALLMARK_GLYCOLYSIS & 187 & -0.444271712 & -1.507184662 & 0.001492077 & 0.002763106 & 0.001047072 & 4567 & tags $=40 \%$, list $=19 \%$, signal $=32 \%$ \\
\hline HALLMARK_XENOBIOTIC_METABOLISM & 189 & -0.434517696 & -1.476402142 & 0.001755408 & 0.003134656 & 0.00118787 & 3909 & tags $=33 \%$, list $=16 \%$, signal $=28 \%$ \\
\hline HALLMARK_WNT_BETA_CATENIN_SIGNALING & 41 & 0.536388636 & 1.74398709 & 0.002325471 & 0.004009434 & 0.001519364 & 1498 & tags $=29 \%$, list $=6 \%$, signal $=28 \%$ \\
\hline HALLMARK_ANDROGEN_RESPONSE & 91 & -0.50101427 & -1.581007544 & 0.002826262 & 0.004710437 & 0.001785008 & 2800 & tags $=33 \%$, list $=11 \%$, signal $=29 \%$ \\
\hline HALLMARK_ALLOGRAFT_REJECTION & 186 & -0.413677412 & -1.401314341 & 0.009797813 & 0.015802924 & 0.005988476 & 4587 & tags $=28 \%$, list $=19 \%$, signal $=23 \%$ \\
\hline HALLMARK_PI3K_AKT_MTOR_SIGNALING & 98 & -0.453102918 & -1.444475416 & 0.010245909 & 0.016009233 & 0.006066657 & 5144 & tags $=44 \%$, list $=21 \%$, signal $=35 \%$ \\
\hline
\end{tabular}

NMIBC, non-muscle-invasive bladder cancer. 